\title{
On inverse problems for semiconductor equations
}

\author{
$\begin{array}{lll}\text { M. Burger } & & \text { H.W. Engl } \\ & \text { A. Leitão } & \text { P.A. Markowich } \\ & 2,3\end{array}$
}

November 24, 2020

${ }^{1}$ Institut für Industriemathematik, Johannes Kepler Universität, A-4040 Linz, Austria (engl@indmath.uni-linz.ac.at)

2 Johann Radon Institute for Computational and Applied Mathematics, Austrian Academy of Sciences c/o Johannes Kepler Universität, A-4040 Linz, Austria (antonio.leitao@oeaw.ac.at)

${ }^{3}$ Institut für Mathematik, Universität Wien, Boltzmanngasse 9, A-1090 Vienna, Austria (peter.markowich@univie.ac.at)

\begin{abstract}
This paper is devoted to the investigation of inverse problems related to stationary drift-diffusion equations modeling semiconductor devices. In this context we analyze several identification problems corresponding to different types of measurements, where the parameter to be reconstructed is an inhomogeneity in the PDE model (doping profile).

For a particular type of measurement (related to the voltage-current map) we consider special cases of drift-diffusion equations, where the inverse problems reduces to a classical inverse conductivity problem. A numerical experiment is presented for one of these special situations (linearized unipolar case).
\end{abstract}

\section{Introduction}

The drift diffusion equations are the most widely used model to describe semiconductor devices. From the point of view of applications, there is great interest in replacing laboratory testing by numerical simulation in order to minimize development costs. For the current state of technology, the drift diffusion equations represent a realistic compromise between computational efficiency (to solve this nonlinear system of partial differential equations) and an accurate description of the underlying device physics.

The name drift diffusion equations of semiconductors originates from the type of dependence of the current densities on the carrier densities and the electric field. The current densities are the sums of drift terms and diffusion terms. It is worth mentioning that, with the increased miniaturization of semiconductor devices, one comes closer and closer to the limits of validity of the drift diffusion 
equation. This is due to the fact that in ever smaller devices the assumption that the free carriers can be modeled as a continuum becomes invalid. On the other hand, the drift diffusion equations are derived by a scaling limit process, where the mean free path of a particle tends to zero.

The mathematical modeling of semiconductor equations has developed significantly, together with their manufacturing. The basic semiconductor device equations where first presented, in the level of completeness described in this paper, by W. Van Roosbroeck (see [56]) in 1950. Since then they have been subject of intensive mathematical and numerical investigation (cf. 42 for an overview).

This paper is devoted to the investigation of inverse problems related to stationary drift-diffusion equations modeling semiconductor devices. In this context we analyze several inverse problems related to the identification of doping profiles. In all these inverse problems the parameter to be identified corresponds to the so called doping profile (a parameter function in a system of PDE's). However, the reconstruction problems are related to data generated by different types of measurement techniques.

The paper is organized as follows. In section 2 we describe the stationary and transient drift diffusion equations. Some existence and uniqueness results (needed further in the text) are presented and some particular models (derived from different simplification assumptions) are investigated. In Section 3 the inverse doping problems are presented. We address the inverse problems modeled by the voltage-current map, by capacitance measurements, and by Laser-beaminduced measurements. We also address the identification issue for some of the inverse problems mentioned above. In Section 4 we present some new numerical results for an identification problem related to the voltage-current map (linearized unipolar case). The results are obtained using the Landweber-Kaczmarz method.

There are other relevant inverse problems for semiconductor equations that are not covered in this paper:

- The inverse problem of identifying transistor contact resistivity of planar electronic devices, such as MOSFETs (metal oxide semiconductor fieldeffect transistors) is treated in 22 . It is shown that a one-point boundary measurement of the potential is sufficient to identify the resistivity from a one-parameter monotone family, and such identification is both stable and continuously dependent on the parameter. Because of the device miniaturization, it is impossible to measure the contact resistivity in a direct way to satisfactory accuracy. There are extensive experimental and simulation studies for the determination of contact resistivity by certain accessible boundary measurements.

- A similar problem of determining the contact resistivity of a semiconductor device from a single voltage measurement is investigated in [15]. It can be modeled as an inverse problem for the elliptic differential equation $\Delta V-p \chi(S) u=0$ in $\Omega \subset \mathcal{R}^{2}, \partial V / \partial n=g \geq 0$ but $g \not \equiv 0$ on $\partial \Omega$, where $V(x)$ 
is the measured voltage, $S \subset \Omega$ and $p>0$ are unknown. In this paper, the authors consider the identification of $p$ when the contact location $S$ is also known.

- The problem of optimal design of devices, where the aim is to find a doping profile that can reach certain design goals, e.g., maximum drive current while keeping the leakage current below a certain threshold. From a computational point of view, this problem exhibits many similarities to the inverse doping problems considered in this paper, with the difference that in optimal design one usually has to solve the drift-diffusion equations for only one or two different applied voltages. We refer to [14, 32, 54

\section{Semiconductor equations}

The basic semiconductor device equations consist of the Poisson equation (1), the continuity equations for electrons (2) and holes (3), and the current relations for electrons (4) and holes (5). For some applications, in order to account for thermal effects in semiconductor devices, its also necessary to add to this system the heat flow equation (6).

$$
\begin{aligned}
& \operatorname{div}(\epsilon \nabla V)=q(n-p-C) \\
& \operatorname{div} J_{n}=q\left(\partial_{t} n+R\right) \\
& \operatorname{div} J_{p}=q\left(-\partial_{t} p-R\right) \\
& J_{n}=q\left(D_{n}(E, T) \nabla n-\mu_{n}(E, T) n \nabla V\right) \\
& J_{p}=q\left(-D_{p}(E, T) \nabla p-\mu_{p}(E, T) p \nabla V\right) \\
& \rho c(T) \partial_{t} T-H=\operatorname{div} k(T) \nabla T,
\end{aligned}
$$

defined in a domain $\Omega \subset \mathbb{R}^{d}(d=1,2,3)$ representing the semiconductor device. Here $V$ denotes the electrostatic potential $(-\nabla V$ is the electric field, $E=|\nabla V|), n$ and $p$ are the concentration of free carriers of negative charge (electrons) and positive charge (holes) respectively, and $J_{n}$ and $J_{p}$ are the densities of the electron and the hole current respectively. $D_{n}$ and $D_{p}$ are the diffusion coefficients for electrons and holes respectively. $\mu_{n}$ and $\mu_{p}$ represent the mobilities of electrons and holes respectively. The positive constants $\epsilon$ and $q$ denote the permittivity coefficient (for silicon) and the elementary charge respectively. The function $R=R(n, p, x)$ denotes the recombination-generation rate. The function $C=C(x)$ represent the doping concentration, which is produced by diffusion of different materials into the silicon crystal and by implantation with an ion beam. The constants $\rho$ and $c$ represent the specific mass density and specific heat of the material. $k$ and $H$ denote the thermal conductivity and the locally generated heat.

This set of equations is considered in a domain $\Omega \subset \mathbb{R}^{d}(d=1,2,3)$ representing the semiconductor device. We assume the boundary $\partial \Omega$ of $\Omega$ to be divided into two nonempty disjoint parts: $\partial \Omega=\overline{\partial \Omega_{N}} \cup \overline{\partial \Omega_{D}}$. The Dirichlet part of the boundary $\partial \Omega_{D}$ models the Ohmic contacts, where the potential $V$ 
as well as the concentrations $n$ and $p$ are prescribed. The Neumann part $\partial \Omega_{N}$ of the boundary corresponds to insulating surfaces, thus a zero current flow and a zero electric field in the normal direction are prescribed.

In the next subsection, when we turn our attention to the stationary driftdiffusion equations, we shall discuss in more detail the system (1)-(6) as well as corresponding boundary conditions. Detailed expositions of the subject of modeling, analysis and simulation of semiconductor equations can be found in the books of S. Selberherr [53, P. Markowich 44] and P. Markowich et al [42.

\subsection{Stationary drift diffusion equation}

We shall consider system (1) - (6) under the following assumptions: Thermal effects will not be taken into account, i.e. we shall work under the assumption of constant particle temperature. Further, we consider the carrier concentrations $n$ and $p$ and the potential $V$ as time-independent functions.

Under the above assumptions, if we substitute the current relations for electrons and holes (4) and (5) into the corresponding continuity equations (2) and (3), we obtain a coupled system of partial differential equations, the so called stationary drift diffusion equation:

$$
\begin{aligned}
\operatorname{div}\left(\epsilon_{s} \nabla V\right) & =q(n-p-C), \text { in } \Omega \\
\operatorname{div}\left(D_{n} \nabla n-\mu_{n} n \nabla V\right) & =R, \text { in } \Omega \\
\operatorname{div}\left(D_{p} \nabla p-\mu_{p} p \nabla V\right) & =R, \text { in } \Omega .
\end{aligned}
$$

Next we briefly discuss the modeling of the recombination-generation rate. The bandgap is relatively large for semiconductors (gap between valence and conduction band), and a significant amount of energy is necessary to transfer electrons from the valence and to the conduction band. This process is called generation of electron-hole pairs. On the other hand, the reverse process corresponds to the transfer of a conduction electron into the lower energetic valence band. This process is called recombination of electron-hole pairs. In our model these phenomena are described by the recombination-generation rate $R$. Various models can be found in the literature (see, e.g., 53]). For the sake of simplicity, we shall consider either the Shockley Read Hall rate

$$
R_{S R H}=\frac{n p-n_{i}^{2}}{\tau_{p}\left(n+n_{i}\right)+\tau_{p}\left(p+n_{i}\right)}
$$

or the Auger recombination-generation rate

$$
R_{A U}=\left(C_{n} n+C_{p} p\right)\left(n p-n_{i}^{2}\right),
$$

where $n_{i}$ denotes the intrinsic density, $\tau_{n}$ and $\tau_{p}$ are the lifetimes of electrons and holes respectively (see Table 1 for some typical values for recombination generation parameters). In both cases we can write

$$
R=\mathcal{R}(n, p, x)\left(n p-n_{i}^{2}\right) .
$$




\begin{tabular}{cl}
\hline Parameter & \multicolumn{1}{c}{ Typical value } \\
\hline$\epsilon_{s}$ & $11.9 \epsilon_{0}$ \\
$\mu_{n}$ & $\approx 1500 \mathrm{~cm}^{2} \mathrm{~V}^{-1} \mathrm{~s}^{-1}$ \\
$\mu_{p}$ & $\approx 450 \mathrm{~cm}^{2} \mathrm{~V}^{-1} \mathrm{~s}^{-1}$ \\
$C_{n}$ & $2.8 \times 10^{-31} \mathrm{~cm}^{6} / \mathrm{s}$ \\
$C_{p}$ & $9.9 \times 10^{-32} \mathrm{~cm}^{6} / \mathrm{s}$ \\
$\tau_{n}$ & $10^{-6} \mathrm{~s}$ \\
$\tau_{p}$ & $10^{-5} \mathrm{~s}$ \\
\hline
\end{tabular}

Table 1: Properties of silicon at room temperature (physical constants: Permittivity in vacuum $\epsilon_{0}=8.85 \times 10^{-14} \mathrm{As} \mathrm{V}^{-1} \mathrm{~cm}^{-1}$; Elementary charge $q=$ $\left.1.6 \times 10^{-19} \mathrm{As}\right)$.

Now we shall introduce the boundary conditions. As already mentioned, the boundary $\partial \Omega$ of $\Omega$ is divided in two nonempty parts: $\partial \Omega=\partial \Omega_{N} \cup \partial \Omega_{D}$. Due to the thermal equilibrium assumption it follows $n p=n_{i}^{2}$, and the assumption of vanishing space charge density gives $n-p-C=0$, for $x \in \partial \Omega_{D}$. On the Dirichlet part of the boundary this implies the following type of boundary conditions:

$$
\begin{array}{ll}
V=V_{D}(x):=U(x)+V_{\mathrm{bi}}(x) & \text { on } \partial \Omega_{\mathrm{D}} \\
n=n_{D}(x):=\frac{1}{2}\left(C(x)+\sqrt{C(x)^{2}+4 n_{i}^{2}}\right) & \text { on } \partial \Omega_{\mathrm{D}} \\
p=p_{D}(x):=\frac{1}{2}\left(-C(x)+\sqrt{C(x)^{2}+4 n_{i}^{2}}\right) & \text { on } \partial \Omega_{\mathrm{D}},
\end{array}
$$

where $U(x)$ is the applied potential, (differences in $U(x)$ between different segments of $\partial \Omega_{D}$ correspond to the applied bias between these two contacts), $V_{\mathrm{bi}}(x):=U_{T} \ln \left(\frac{n_{D}(x)}{n_{i}}\right)$ and $U_{T}$ is the thermal voltage.

Since the Neumann part of the boundary $\partial \Omega_{N}=\partial \Omega-\partial \Omega_{D}$ models insulating or artificial surfaces, a zero current flow and a zero electric field in the normal direction are prescribed. Thus, the following homogeneous boundary conditions are supplied (in terms of $J_{n}$ and $J_{p}$ ):

$$
\begin{aligned}
\nabla V \cdot \nu & =0 \text { on } \partial \Omega_{\mathrm{N}} \\
J_{n} \cdot \nu & =0 \text { on } \partial \Omega_{\mathrm{N}} \\
J_{p} \cdot \nu & =0 \text { on } \partial \Omega_{\mathrm{N}} .
\end{aligned}
$$

Next we briefly address the modeling of the doping profile. The function $C(x)$ models a preconcentration of ions in the crystal, so $C(x)=C_{+}(x)-$ $C_{-}(x)$ holds, where $C_{+}$and $C_{-}$are concentrations of negative and positive ions respectively. In those subregions of $\Omega$ in which the preconcentration of negative ions predominate (P-regions), we have $C(x)<0$. Analogously, we define the N-regions, where $C(x)>0$ holds. The boundaries between the $\mathrm{P}$ - and N-regions 


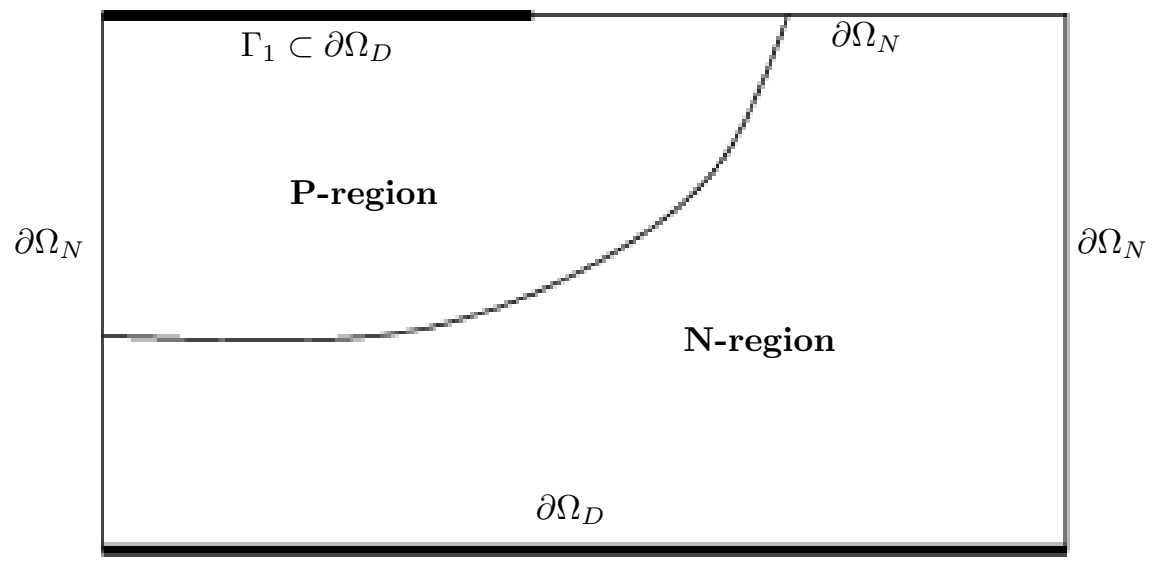

Figure 1: P-N diode. Example of P-N junction.

(where $C$ change sign) are called $\mathrm{P}-\mathrm{N}$ junctions. An example of a device with a very simple $\mathrm{P}-\mathrm{N}$ junction is shown in Figure 1, where a two-dimensional P-N diode is represented.

Now we introduce an important special change of variables, in order to rewrite system (7)- 9 ) as well as boundary conditions $10-12$ and $13-15$ in a more convenient way. This variable transformation is (partially) motivated by the Einstein relations

$$
D_{n}=U_{T} \mu_{n}, \quad D_{p}=U_{T} \mu_{p},
$$

which are a standard assumption about the mobilities and diffusion coefficients. The so called Slotboom variables $(u, v)$ are defined by the relations

$$
n(x)=n_{i} \exp \left(\frac{V(x)}{U_{T}}\right) u(x), \quad p(x)=n_{i} \exp \left(\frac{-V(x)}{U_{T}}\right) v(x) .
$$

For convenience, we rescale the potential and the mobilities:

$$
V(x) \leftarrow V(x) / U_{T}, \quad \tilde{\mu}_{n}:=q U_{T} \mu_{n}, \quad \tilde{\mu}_{p}:=q U_{T} \mu_{p} .
$$

Note that the current relations now read:

$$
J_{n}=\tilde{\mu}_{n} n_{i} e^{V} \nabla u, \quad J_{p}=-\tilde{\mu}_{p} n_{i} e^{-V} \nabla v .
$$

Therefore, we can write the stationary drift diffusion equation in the form 


$$
\begin{aligned}
\lambda^{2} \Delta V & =\delta^{2}\left(e^{V} u-e^{-V} v\right)-C(x), & & \text { in } \Omega \\
\operatorname{div} J_{n} & =\delta^{4} Q(V, u, v, x)(u v-1), & & \text { in } \Omega \\
\operatorname{div} J_{p} & =-\delta^{4} Q(V, u, v, x)(u v-1), & & \text { in } \Omega \\
V & =V_{D}=U+V_{\mathrm{bi}}, & & \text { on } \partial \Omega_{\mathrm{D}} \\
u & =u_{D}=e^{-U,} & & \text { on } \partial \Omega_{\mathrm{D}} \\
v & =v_{D}=e^{U}, & & \text { on } \partial \Omega_{\mathrm{D}} \\
\nabla V \cdot \nu & =0 & & \text { on } \partial \Omega_{\mathrm{N}} \\
J_{n} \cdot \nu & =0 & & \text { on } \partial \Omega_{\mathrm{N}} \\
J_{p} \cdot \nu & =0 & & \text { on } \partial \Omega_{\mathrm{N}},
\end{aligned}
$$

where $\lambda^{2}:=\epsilon /\left(q U_{T}\right), \delta^{2}:=n_{i}$ and the function $Q$ is defined implicitly by the relation $Q(V, u, v, x)=\mathcal{R}(n, p, x)$. Notice the applied potential has also to be rescaled: $U(x) \leftarrow U(x) / U_{T}$.

\subsection{Some existence and uniqueness results for stationary drift diffusion equation}

In this subsection we discuss the solution theory for the system of drift diffusion equations (17)-25). First we present an existence result, which can be found in the monography [42, Theorem 3.3.16].

Proposition 1 Let $\kappa>1$ be a constant satisfying

$$
\kappa^{-1} \leq u_{D}(x), v_{D}(x) \leq \kappa, \forall x \in \partial \Omega_{D},
$$

and let $-\infty<C_{m} \leq C_{M}<+\infty$. Then for any $C \in\left\{L^{\infty}(\Omega) ; C_{m} \leq C(x) \leq\right.$ $\left.C_{M}, x \in \Omega\right\}$ the boundary value problem (17)-25) admits a weak solution $(V, u, v) \in\left(H^{1}(\Omega) \cap L^{\infty}(\Omega)\right)^{3}$ satisfying

$$
\kappa^{-1} \leq u(x), v(x) \leq \kappa, \forall x \in \Omega,
$$

furthermore

$$
\begin{aligned}
& V(x) \geq \min \left(\inf _{\partial \Omega_{D}} V_{D}, U_{T} \ln \left[\frac{1}{2 \kappa n_{i}}\left(C_{m}+\left(C_{m}^{2}+4 n_{i}^{2}\right)^{1 / 2}\right)\right]\right), \text { in } \Omega \\
& V(x) \leq \max \left(\sup _{\partial \Omega_{D}} V_{D}, U_{T} \ln \left[\frac{\kappa}{2 n_{i}}\left(C_{M}+\left(C_{M}^{2}+4 n_{i}^{2}\right)^{1 / 2}\right)\right]\right), \text { in } \Omega .
\end{aligned}
$$

Sketch of the proof:

Solving the Poisson equation and the continuity equations (three elliptic mixed boundary value problems), one at a time, it is possible to define an operator in an appropriate $L^{2}$-space, whose fixed point is a weak solution of 17$)-25$. The 
existence of a fixed point is established by the Schauder Fixed point operator, once one proves that the fixed point operator is completely continuous, which is accomplished by the use of standard elliptic theory.

As far as uniqueness of solutions of system (17)-25) is concerned, a corresponding result can be obtained if the applied voltage is small (in the norm of $\left.L^{\infty}\left(\partial \Omega_{D}\right) \cap H^{3 / 2}\left(\partial \Omega_{D}\right)\right)$. The following uniqueness result corresponds to [12, Theorem 2.4].

Proposition 2 Let the voltage $U$ be such that $\|U\|_{L^{\infty}\left(\partial \Omega_{D}\right)}+\|U\|_{H^{3 / 2}\left(\partial \Omega_{D}\right)}$ is sufficiently small. Then system (17)-25) has a unique solution $(V, u, v) \in$ $\left(H^{1}(\Omega) \cap L^{\infty}(\Omega)\right)^{3}$.

Since existence and uniqueness of solutions for system (17)-25) can be guaranteed for small applied voltages only, it is reasonable to consider instead of this system its linearized version around the equilibrium point $U \equiv 0$ instead. We shall follow this approach through a large part of this paper.

Under stronger assumptions on the boundary parts $\partial \Omega_{D}, \partial \Omega_{N}$ as well as on the boundary conditions $V_{D}, u_{D}, v_{D}$, it is even possible to show $H^{2}$-regularity for a solution $(V, u, v)$ of (17)-25). Next we shall discuss a corresponding result; for the proof details we refer to the monography by Markowich [41]. First we have to consider the following assumptions:

A1) $\Omega$ is a bounded domain of class $C^{0,1}$ in $\mathcal{R}^{d}$ and the $(d-1)$-dimensional Lebesgue measure of $\partial \Omega_{D}$ is positive;

A2) The Dirichlet boundary data $\left(V_{D}, u_{D}, v_{D}\right)$ in $\left.20-22\right)$ satisfy

$$
\left(V_{D}, u_{D}, v_{D}\right) \in\left(H^{2}(\Omega)\right)^{3},\left.\left(V_{D}, u_{D}, v_{D}\right)\right|_{\partial \Omega_{D}} \in\left(L^{\infty}\left(\partial \Omega_{D}\right)\right)^{3} .
$$

Furthermore, $\left.\left(V_{D}, u_{D}, v_{D}\right)\right|_{\partial \Omega_{N}}=(0,0,0)$ and there is $U_{+} \geq 0$ such that

$$
e^{-U_{+}} \leq \inf _{\partial \Omega_{D}} u_{D}, \inf _{\partial \Omega_{D}} v_{D} ; \sup _{\partial \Omega_{D}} u_{D}, \sup _{\partial \Omega_{D}} v_{D} \leq e^{U_{+}} ;
$$

A3) The doping profile satisfies $C \in L^{\infty}(\Omega)$;

A4) The function $Q$ in 18,19 is such that $Q(\cdot, \cdot, \cdot, x) \in C^{1}\left(\mathcal{R} \times(0, \infty)^{2}\right)$ for all $x \in \Omega ; Q(V, u, v, \cdot), \nabla_{(V, u, v)} Q(V, u, v, \cdot) \in L^{\infty}(\Omega)$ uniformly for $(V, u, v)$ in bounded sets of $\mathcal{R} \times(0, \infty)^{2} ; Q(V, u, v, x) \geq 0$ in $\mathcal{R} \times(0, \infty)^{2} \times \Omega$;

A5) The mobilities $\mu_{n}, \mu_{p}$ satisfy: $\mu_{n}=\mu_{n}(x), \mu_{p}=\mu_{p}(x), \mu_{n}, \mu_{p} \in W^{1, \infty}(\Omega)$; functions $\mu_{n}, \mu_{p}$ are both positive and uniformly bounded (away from zero) in $\Omega$;

A6) The solution of

$$
\Delta w=f \text { in } \Omega,\left.\quad w\right|_{\partial \Omega_{D}}=\left.w_{\nu}\right|_{\partial \Omega_{N}}=0,
$$

satisfies $\|w\|_{2, q, \Omega} \leq K_{1}\|f\|_{q, \Omega}$ for every $f \in L^{q}(\Omega)$ with $q=2$ and $q=3 / 2$. 
Proposition 3 [41, Theorem 3.3.1] Let's assume that assumptions A1) A6) hold. Then every weak solution $(V, u, v) \in\left(H^{1}(\Omega) \cap L^{\infty}(\Omega)\right)^{3}$ satisfies

$$
(V, u, v) \in\left(H^{2}(\Omega)\right)^{3} .
$$

Notice that, in the 2-dimensional case, assumption A6) can only be satisfied (for $q=2$ ) if the angle between the Neumann and Dirichlet parts of $\partial \Omega$ is smaller than $\pi / 2$. Otherwise, the solutions of the elliptic mixed boundary value problems in A6) will not belong to any space $H^{1+\epsilon}(\Omega), \epsilon>0$ (see [26] for details).

If assumption A6) holds only for $q=2$, it is still possible to prove $H^{2}$ regularity for the solution $V$ of the Poisson equation. However, it is not possible to prove square-integrability of all second derivatives of $u$ and $v$, but this is usually not needed for the formulation of the inverse problem.

\subsection{The equilibrium case}

In this subsection we analyze the equilibrium case for the stationary drift diffusion equations, which corresponds to the assumption $U(x) \equiv 0$. In this particular case several simplifications are possible. It is immediate to see that the solution of 177-25) is of the form $\left(V=V^{0}, u \equiv 1, v \equiv 1\right)$, where

$$
\left\{\begin{aligned}
\lambda^{2} \Delta V^{0} & =e^{V^{0}}-e^{-V^{0}}-C(x) & & \text { in } \Omega \\
V^{0} & =V_{\mathrm{bi}}(x) & & \text { on } \partial \Omega_{D} \\
\nabla V^{0} \cdot \nu & =0 & & \text { on } \partial \Omega_{N} .
\end{aligned}\right.
$$

As already mentioned in subsection 2.2 , we shall be interested in the linearized drift diffusion system at the equilibrium. Keeping this in mind, we compute the derivative of the solution of $177-(25)$ with respect to the voltage $U$ at $U \equiv 0$ in the direction $h$. This directional derivative is given by the solution $(\hat{V}, \hat{u}, \hat{v})$ of

$$
\begin{cases}\lambda^{2} \Delta \hat{V}=e^{V^{0}} \hat{u}+e^{-V^{0}} \hat{v}+\left(e^{V^{0}}+e^{-V^{0}}\right) \hat{V} & \text { in } \Omega \\ \operatorname{div}\left(\mu_{n} e^{V^{0}} \nabla \hat{u}\right)=Q_{0}\left(V^{0}, x\right)(\hat{u}+\hat{v}) & \text { in } \Omega \\ \operatorname{div}\left(\mu_{p} e^{-V^{0}} \nabla \hat{v}\right)=Q_{0}\left(V^{0}, x\right)(\hat{u}+\hat{v}) & \text { in } \Omega \\ \hat{V}=h & \text { on } \partial \Omega_{D} \\ \hat{u}=-h & \text { on } \partial \Omega_{D} \\ \hat{v}=h & \text { on } \partial \Omega_{D} \\ \frac{\partial V^{0}}{\partial \nu}=\frac{\partial \hat{u}}{\partial \nu}=\frac{\partial \hat{v}}{\partial \nu}=0 & \text { on } \partial \Omega_{N},\end{cases}
$$

where $Q_{0}\left(V^{0}, x\right)=Q\left(V^{0}, 1,1, x\right)$. Notice that, in the linearized case close to equilibrium, the solutions $(\hat{u}, \hat{v})$ of the continuity equations do not depend on the electrostatic potential $\hat{V}$. 


\subsection{Unipolar and bipolar cases}

In this subsection we introduce two special cases, which are going to play a key rule in the modeling of some of the inverse problems analyzed in this paper. We start by introducing the operator called voltage-current $(\mathrm{V}-\mathrm{C})$ map:

$$
\begin{aligned}
\Sigma_{C}: H^{3 / 2}\left(\partial \Omega_{D}\right) & \rightarrow H^{1 / 2}\left(\Gamma_{1}\right) \\
U & \left.\mapsto J \cdot \nu\right|_{\Gamma_{1}}=\left.\left(J_{n}+J_{p}\right) \cdot \nu\right|_{\Gamma_{1}},
\end{aligned}
$$

where $\Gamma_{1} \subset \partial \Omega_{D}$ (see Figure 1 for an example). The map $\Sigma_{C}$ takes the applied voltage $U$ into the outflow current density on $\Gamma_{1}$. The linearized unipolar case (close to equilibrium) corresponds to the model obtained from the unipolar drift diffusion equations by linearizing the $\mathrm{V}-\mathrm{C}$ map at $U \equiv 0$. This simplification is motivated by the fact that the $\mathrm{V}-\mathrm{C}$ map can only be defined as a single-valued function in a neighborhood of $U=0$. Furthermore, the following assumptions are also taken into account

i) The concentration of holes satisfy $p=0$ (or, equivalently, $v=0$ in $\Omega$ );

ii) No recombination-generation rate is present, i.e. $\mathcal{R}=0$ (or $Q=0$ ).

Under this assumptions, system (17)-25 reduces to the decoupled system:

$$
\begin{cases}\lambda^{2} \Delta V^{0}=e^{V^{0}}-C(x) & \text { in } \Omega \\ \operatorname{div}\left(e^{V^{0}} \nabla \hat{u}\right)=0 & \text { in } \Omega \\ V^{0}=V_{\mathrm{bi}}(x) & \text { on } \Omega_{D} \\ \hat{u}=U(x) & \text { on } \Omega_{D} \\ \nabla V^{0} \cdot \nu=0 & \text { on } \Omega_{N} \\ \hat{J}_{n} \cdot \nu=0 & \text { on } \Omega_{N}\end{cases}
$$

The inverse problem of identifying the doping profile in the linearized unipolar model 28) corresponds to identification of $C(x)$ from the map

$$
\Sigma_{C}^{\prime}(0):\left.U \mapsto\left(\hat{J}_{n} \cdot \nu\right)\right|_{\Gamma_{1}}, \quad \hat{J}_{n}:=\mu_{n} e^{V_{0}} \nabla \hat{u} .
$$

Notice that, since $V=V_{\mathrm{bi}}$ is known at $\partial \Omega_{D}$, the current data (output) $J_{n} \cdot \nu=$ $\mu_{n} e^{V^{0}} u_{\nu}$ can be directly replaced by the Neumann data $u_{\nu}$.

We shall return to this identification problem in Section 3 , where the inverse problem described above is considered as a generalization of the well known electrical impedance tomography or inverse conductivity problem (see, e.g., 9, 35] for a survey on these inverse problems).

Next we concentrate on deriving the so called bipolar case. As in the unipolar case, we will be interested in reconstructing the doping profile $C$ in 17$)-(25)$ from the linearized $\mathrm{V}-\mathrm{C}$ map at $U \equiv 0$. This is an interesting case, due to the fact that the Poisson Equation and the continuity equations decouple.

From (27) we see that the Gateaux derivative of the V-C map $\Sigma_{C}$ at the point $U=0$ in the direction $\Phi$ is given by the expression

$$
\Sigma_{C}^{\prime}(0) \Phi:=\left.\left(\mu_{n} e^{V_{\mathrm{bi}}} \hat{u}_{\nu}-\mu_{p} e^{-V_{\mathrm{bi}}} \hat{v}_{\nu}\right)\right|_{\Gamma_{1}}
$$


where $(u, v)$ solve

$$
\begin{cases}\operatorname{div}\left(\mu_{n} e^{V^{0}} \nabla \hat{u}\right)=Q_{0}\left(V^{0}, x\right)(\hat{u}+\hat{v}) & \text { in } \Omega \\ \operatorname{div}\left(\mu_{p} e^{-V^{0}} \nabla \hat{v}\right)=Q_{0}\left(V^{0}, x\right)(\hat{u}+\hat{v}) & \text { in } \Omega \\ \hat{u}=-\Phi & \text { on } \partial \Omega_{D} \\ \hat{v}=\Phi & \text { on } \partial \Omega_{D} \\ \frac{\partial \hat{u}}{\partial \nu}=\frac{\partial \hat{v}}{\partial \nu}=0 & \text { on } \partial \Omega_{N}\end{cases}
$$

and $V^{0}$ is the solution of the equilibrium problem (26).

Notice that the solution of the Poisson equation can be computed a priori, since it does not depend on $\Phi$. The linear operator $\Sigma_{C}^{\prime}(0)$ is continuous. Actually we can prove more: since $(u, v)$ depend continuously (in $\left.H^{2}(\Omega)^{2}\right)$ on the boundary data $\Phi$ (in $H^{3 / 2}\left(\partial \Omega_{D}\right)$ ), it follows from the boundedness and compactness of the trace operator $\gamma: H^{2}(\Omega) \rightarrow H^{1 / 2}\left(\Gamma_{1}\right)$ that $\Sigma_{C}^{\prime}(0)$ is a bounded and compact operator. The application $\Sigma_{C}^{\prime}(0)$ maps the Dirichlet data for $(u, v)$ to a weighted sum of their Neumann data and can be compared with the identification problem in the electrical impedance tomography.

\subsection{Flipped bipolar case}

In this subsection we introduce another special case, which will be relevant for the formulation of the inverse problem related to the laser-beam-induced current (LBIC) measurements. We start by introducing the LBIC functional defined by the boundary integral

$$
\begin{aligned}
\mathcal{I}: L^{2}(\Omega) & \rightarrow \mathcal{R} \\
g & \mapsto \int_{\Gamma_{1}}\left\{\mu_{n} e^{V^{0}} \hat{u}_{\nu}-\mu_{p} e^{-V^{0}} \hat{v}_{\nu}\right\} d s
\end{aligned}
$$

where $\Gamma_{1} \subset \partial \Omega_{D}$ is defined as in Subsection 2.4. $V^{0}$ is the solution of the equilibrium problem (26) and $(u, v)$ solve

$$
\begin{cases}\operatorname{div}\left(\mu_{n} e^{V^{0}} \nabla \hat{u}\right)=Q_{0}\left(V^{0}, x\right)(\hat{u}+\hat{v})+g & \text { in } \Omega \\ \operatorname{div}\left(\mu_{p} e^{-V^{0}} \nabla \hat{v}\right)=Q_{0}\left(V^{0}, x\right)(\hat{u}+\hat{v})+g & \text { in } \Omega \\ \hat{u}=\hat{v}=0 & \text { on } \partial \Omega_{D} \\ \frac{\partial \hat{u}}{\partial \nu}=\frac{\partial \hat{v}}{\partial \nu}=0 & \text { on } \partial \Omega_{N}\end{cases}
$$

Notice that the only differences between systems $(30)$ and $\sqrt{29})$ (from the bipolar case) are: 1) the $L^{2}(\Omega)$ function $g$, appearing on the right hand side of the linearized continuity equations and representing the applied laser beam (see Section 3 for details on the problem formulation); 2) the Dirichlet boundary condition at $\partial \Omega_{D}$.

The inverse problem of reconstructing the doping profile from measurements of the LBIC functional was considered in 23, 24]. An alternative representation for the functional $\mathcal{I}$ was derived in 23$]$. Analyzing the variational formulation of 
the system constituted by (26) and (30) and using standard functional analytical arguments as well as basic elliptic theory (see [28]), the authors proved that the LBIC functional can be written as

$$
\mathcal{I}(g)=\hat{\langle} v-\tilde{u}, g\rangle_{L^{2}(\Omega)},
$$

where $V^{0}$ is defined as before and $(\hat{u}, \hat{v})$ solve the system

$$
\begin{cases}\operatorname{div}\left(\mu_{n} e^{V^{0}} \nabla \tilde{u}\right)=Q_{0}\left(V^{0}, x\right)(\tilde{u}-\tilde{v}) & \text { in } \Omega \\ \operatorname{div}\left(\mu_{p} e^{-V^{0}} \nabla \tilde{v}\right)=Q_{0}\left(V^{0}, x\right)(\tilde{v}-\tilde{\tilde{u}}) & \text { in } \Omega \\ \tilde{u}=\tilde{v}=1 & \text { on } \Gamma_{1} \\ \tilde{u}=\tilde{v}=0 & \text { on } \partial \Omega_{D} / \Gamma_{1} \\ \frac{\partial \tilde{u}}{\partial \nu}=\frac{\partial \tilde{u}}{\partial \nu}=0 & \text { on } \partial \Omega_{N}\end{cases}
$$

We shall refer to system (26), (32) as flipped bipolar case. As in the bipolar case, the solution of the Poisson equation can be computed a priori, since $V^{0}$

does not depend on $g$. Therefore, to evaluate $\mathcal{I}$ in (31) one needs only to solve the coupled system (32). Moreover, from the representation formula (31), it follows that $\mathcal{I}$ is a linear continuous functional on $L^{2}(\Omega)$.

\section{Inverse doping problem}

The so called inverse doping profile corresponds to the problem of identifying a doping profile $C(x)$ in system (17)-(25) from indirect measurements. In practical applications, the following types of measurements are available (cf. [38):

1. Current flow through a contact $\Gamma_{1} \subset \partial \Omega_{D}$ :

$$
I(U)=\int_{\Gamma_{1}}\left(J_{n}+J_{p}\right) \cdot \nu d s,
$$

where $U \in H^{3 / 2}\left(\partial \Omega_{D}\right)$ with $\|U\|$ small.

Under the (idealized, but technologically realizable) assumption that we not only know the averaged flow through $\Gamma_{1}$, but the actual flow $J \cdot \nu$ on $\Gamma_{1}$, this type of measurement corresponds to the voltage-current map introduced in Subsection 2.4

$$
\Sigma_{C}(U):=\left.\left(J_{n}+J_{p}\right) \cdot \nu\right|_{\Gamma_{1}} \in H^{1 / 2}\left(\Gamma_{1}\right) .
$$

2. Mean capacitance of a contact $\Gamma_{1} \subset \partial \Omega_{D}$ :

$$
C a p(U)=\frac{\partial}{\partial U}\left(\int_{\Gamma_{1}} \nabla V \cdot \nu d s\right) .
$$

We shall consider the idealized (but again technologically realizable) data corresponding to measurements of the variation of the electric flux (in the 
normal outward direction) with respect to an applied voltage $U$ at $\partial \Omega_{D}$. This data corresponds to the so called capacitance measurements

$$
\mathcal{T}_{C}(U):=\left.\frac{\partial}{\partial U} \frac{\partial V}{\partial \nu}\right|_{\Gamma_{1}}=\left.\frac{\partial \hat{V}}{\partial \nu}\right|_{\Gamma_{1}} \in H^{1 / 2}\left(\Gamma_{1}\right),
$$

here $V$ is the solution of the Poisson equation for an applied voltage $U \in$ $H^{3 / 2}\left(\partial \Omega_{D}\right)$.

3. Measurements of the total current $i(x)$ flowing out through one contact induced by a laser beam applied at different locations $x \in \Omega$ :

$$
i(x):=\mathcal{I}(\delta(\cdot-x))=\hat{v}(x)-\hat{u}(x),
$$

where $\mathcal{I}$ is the LBIC functional defined in Subsection 2.5 and $(\hat{u}, \hat{v})$ is the solution of system (32). These data correspond to the so called laserbeam-inducted current measurements.

In all cases we assume that $\Gamma_{1} \subset \partial \Omega_{D}$ is sufficiently regular with non zero measure. The first step in the investigation of the inverse problems modeled by operators $\Sigma_{C}$ and $\mathcal{T}_{C}$ consists in analyzing whether these operators are well defined in appropriate spaces. The next three subsections are devoted to the analysis of each of these operators. In the last subsection we discuss in details the inverse problem related to the $\mathrm{V}-\mathrm{C}$ map for the linearized unipolar case close to equilibrium and its relation with the electrical impedance tomography.

\subsection{The voltage-current map}

in this subsection we analyze the $\mathrm{V}-\mathrm{C}$ map introduced above. The map $\Sigma_{C}$ takes (for a fixed doping profile $C$ ) the applied voltage $U$ into the corresponding current density. The non-linear operator $\Sigma_{C}$ is well-defined, when considered as a map between suitable Sobolev spaces. This assertion is a consequence of the following result:

Proposition 4 [12, Proposition 3.1] For each applied voltage $U \in B_{r}(0) \subset$ $H^{3 / 2}\left(\partial \Omega_{D}\right)$ with $r>0$ sufficiently small, the current $J \cdot \nu \in H^{1 / 2}\left(\Gamma_{1}\right)$ is uniquely defined. Furthermore, $\Sigma_{C}: H^{3 / 2}\left(\partial \Omega_{D}\right) \rightarrow H^{1 / 2}\left(\Gamma_{1}\right)$ is continuous and is continuously differentiable in $B_{r}(0)$.

Sketch of the proof:

The first part of the proof follows basically from the uniqueness of solutions for system (17)-25) in $H^{2}(\Omega)^{3}$ together with regularity properties of the Neumann trace operator $\gamma: H^{2}(\Omega) \rightarrow H^{1 / 2}\left(\Gamma_{1}\right)$. The Fréchet-differentiability follows from standard estimates of the residual in the Taylor expansion of the operator $\Sigma_{C}$.

By iterating the argumentation in Proposition 4, one can even prove that $\Sigma_{C}$ is of class $C^{\infty}$ in $B_{r}(0) \subset H^{3 / 2}\left(\partial \Omega_{D}\right)$ for $r$ sufficiently small. 
Proposition 4 establishes a basic property to consider the inverse problem of reconstructing the doping profile $C$ from the $\mathrm{V}-\mathrm{C}$ map. In the sequel we shall consider two possible inverse problems for the $\mathrm{V}-\mathrm{C}$ map.

In the first inverse problem we assume that, for each $C$, the output corresponds to the map $\Sigma_{C}$. A realistic experiment corresponds to measure, for given $\left\{U_{j}\right\}_{j=1}^{N}$, with $\left\|U_{j}\right\|=1$, the outputs

$$
\left\{\Sigma_{C}\left(t U_{j}\right) \mid j=1, \cdots, N ; \quad t \in[0, r]\right\} .
$$

This data corresponds to the assumption that the amplitude of an applied voltage $U_{j}$ can be varied continuously in a practical experiment. In practice, the functions $U_{j}$ are chosen to be piecewise constant.

Notice that, for fixed $U_{j}$, the continuity of $\Sigma_{C}$ implies the continuity of the function $t \mapsto \Sigma_{C}\left(t U_{j}\right)$. Therefore, we have

$$
\Sigma_{C}\left(\cdot U_{j}\right) \in C\left(0, r ; H^{1 / 2}\left(\Gamma_{1}\right)\right) \subset L^{2}\left(0, r ; L^{2}\left(\Gamma_{1}\right)\right) .
$$

This allow the following abstract formulation of the inverse problem for the $\mathrm{V}-\mathrm{C}$ map:

$$
F(C)=Y,
$$

where

1) Parameter: $C=C(x) \in L^{2}(\Omega)=: \mathcal{X}$;

2) Output: $Y=\left\{\Sigma_{C}\left(\cdot U_{j}\right)\right\}_{j=1}^{N} \in\left[L^{2}\left((0, r) \times \Gamma_{1}\right)\right]^{N}=: \mathcal{Y}$;

3) Parameter-to-output map: $F: \mathcal{X} \rightarrow \mathcal{Y}$.

The domain of definition of the operator $F$ is

$$
D(F):=\left\{C \in L^{2}(\Omega) ; C_{m} \leq C(x) \leq C_{M}, \text { a.e. in } \Omega\right\},
$$

where $\underline{C}$ and $\bar{C}$ are appropriate positive constants.

This choice of spaces is motivated by Propositions 2 and 3 , which guarantee, for each $t\left\|U_{j}\right\|<r(r$ small $)$ and $C \in D(F)$, the existence and uniqueness of a $H^{2}$-solution $(V, n, p)$ for system 17 - 25). Therefore, the map

$$
\begin{aligned}
F: D(F) \subset \mathcal{X} & \rightarrow \mathcal{Y} \\
C & \mapsto\left\{\Sigma_{C}\left(\cdot U_{j}\right)\right\}_{j=1}^{N}
\end{aligned}
$$

is well defined. Furthermore, $F$ is also Fréchet-differentiable in $D(F)$. Indeed, we already know that the map $\left.(V, u, v) \mapsto J \cdot \nu\right|_{\Gamma_{1}}$ is continuously differentiable (this is included in the proof of Proposition 4). Thus, it is enough to verify the differentiability of the map $\mathcal{U}_{j}: D(F) \ni C \mapsto(V, u, v) \in H^{2}(\Omega)^{3}$, for fixed $U_{j}$. The variation of the solution $(V, u, v)$ of system $\left.(17)-25\right)$ with respect to a variation of the doping profile $C$ can be deduced similarly as in $(27)$. To prove Fréchet-differentiability of $\mathcal{U}_{J}$, we only have to estimate the residual in the Taylor expansion of this map, as in the proof of Proposition 4 
This inverse problem is addressed in the literature as identification of doping profiles from full voltage-current data. Next we shall investigate a different formulation of the same inverse problem related to the $\mathrm{V}-\mathrm{C}$ map.

In practical applications, the $\mathrm{V}-\mathrm{C}$ map can only be defined in a neighborhood of $U=0$ (due to hysteresis defects for large applied voltages). This motivates the analysis of the problem of identifying the doping profile $C$ from the linearized $\mathrm{V}-\mathrm{C}$ map at $U=0$. (see unipolar and bipolar cases in Subsection 2.4).

As described in Subsection 2.4 the Gateaux derivative of the $\mathrm{V}-\mathrm{C}$ map at $U=0$ in direction $\Phi$ is given by

$$
\Sigma_{C}^{\prime}(0) \Phi=\left.\left(\mu_{n} e^{V_{\mathrm{bi}}} u_{\nu}-\mu_{p} e^{-V_{\mathrm{bi}}} v_{\nu}\right)\right|_{\Gamma_{1}},
$$

where $(u, v)$ solve the system in 29$)$ and $V^{0}$ is the solution of the equilibrium case (26). In Subsection 2.4 we have already verified the boundedness and compactness of $\Sigma_{C}^{\prime}(0)$. Contrary to the case of full data, the solution of the Poisson equation can be computed a priori, since it is independent of $\Phi$.

The next step to complete the setup of this second inverse problem for the $\mathrm{V}-\mathrm{C}$ map is to define the problem data. The data for the problem can be obtained from the full $\mathrm{V}-\mathrm{C}$ data:

$$
Y:=\left\{\Sigma_{C}^{\prime}(0) U_{j}\right\}_{j=1}^{N} \in\left[L^{2}\left(\Gamma_{1}\right)\right]^{N} .
$$

In the literature, this inverse problem in called identification of doping profiles from reduced voltage-current data. Notice that the functions $U_{j}$ are defined as before. Therefore, we obtain for the inverse problem with reduced data the same abstract formulation as in 33 with

1) Parameter: $C=C(x) \in L^{2}(\Omega)=: \mathcal{X}$;

2) Output: $Y=\left\{\Sigma_{C}^{\prime}\left(\cdot U_{j}\right)\right\}_{j=1}^{N} \in\left[L^{2}\left(\Gamma_{1}\right)\right]^{N}=: \mathcal{Y}$;

3) Parameter-to-output map: $F: \mathcal{X} \rightarrow \mathcal{Y}$.

The domain of definition of the operator $F$ is the same as in the case of full data. Notice that the parameter-to-output operator for reduced data is given by:

$$
\begin{aligned}
F: D(F) \subset \mathcal{X} & \rightarrow \mathcal{Y} \\
C & \mapsto\left\{\Sigma_{C}^{\prime}(0) U_{j}\right\}_{j=1}^{N}
\end{aligned}
$$

Analogously as in the full $\mathrm{V}-\mathrm{C}$ data case, one can prove that the non-linear parameter-to-output operator is well defined and Fréchet differentiable in its domain of definition $D(F)$.

As already observed, the solution of the Poisson equation can be computed a priori. The remaining problem (coupled system for $(u, v)$ ) is quite similar to the problem of electrical impedance tomography. In this inverse problem the aim is to identify the conductivity $q=q(x)$ in the equation:

$$
-\operatorname{div}(q \nabla u)=f \text { in } \Omega
$$


from measurements of the Dirichlet-to-Neumann map, which maps the applied voltage $\left.u\right|_{\partial \Omega}$ to the electrical flux $\left.q u_{\nu}\right|_{\partial \Omega}$. The application $\Sigma_{C}^{\prime}(0)$ maps the Dirichlet data for $\hat{u}$ and $\hat{v}$ to the weighted sum of their Neumann data. It can be seen as the counterpart of electrical impedance tomography for common conducting materials.

We close this subsection discussing yet another inverse problem for the capacitance measurements. This problem again concerns the reduced $\mathrm{V}-\mathrm{C}$ map and arrises in a limiting case of the drift diffusion equations, called limit of zero space charge, which is mathematically represented by the scaling limit $\lambda \rightarrow 0$. In this case the Poisson equation reduces to an algebraic relation between $V$ and $C$ and existence of solutions of the zero-space-charge problem in $L^{\infty}(\Omega)$ has been proven in 42 .

Notice that, in the limiting case $\lambda=0$, without further regularity assumptions on the doping profile $C$ we can only guarantee $H^{1}$ regularity for a solution $(u, v)$ of 29$)$. Therefore, $J \cdot \nu \in H^{-1 / 2}\left(\Gamma_{1}\right)$ follows. However, as already observed in [12, if $\nabla C \in L^{p}(\Omega)$ for $p$ sufficiently large $(p \geq 6)$, one can show that the reduced V-C map exists and maps continuously to $L^{2}\left(\Gamma_{1}\right)$.

From the Poisson equation in equilibrium we obtain $\sinh V=2 C$ and the linearized continuity equations can be written in the form

$$
\begin{cases}\operatorname{div}\left(\mu_{n} a \nabla \hat{u}\right)=q(a, x)(\hat{u}+\hat{v}) & \text { in } \Omega \\ \operatorname{div}\left(\mu_{p} a^{-1} \nabla \hat{v}\right)=q(a, x)(\hat{u}+\hat{v}) & \text { in } \Omega \\ u=-\Phi & \text { on } \partial \Omega_{D} \\ v=\Phi & \text { on } \partial \Omega_{D} \\ \frac{\partial u}{\partial \nu}=\frac{\partial v}{\partial \nu}=0 & \text { on } \partial \Omega_{N}\end{cases}
$$

where

$$
a=a(C)=e^{\operatorname{arcsinh}(2 C)}, \quad q(a, x)=Q(\ln (a), 1,1, x) .
$$

Thus, in this limiting case, the inverse doping profile problem reduces to the identification of the conductivity $a$ in the coupled system (34) from the reduced $\mathrm{V}-\mathrm{C}$ map. Once we have reconstructed the coefficient $a$, the doping profile can be obtained from the relation $C=\frac{1}{2} \sinh (\ln a)$.

\subsection{Capacitance measurements}

In this subsection we address the inverse problem modeled by the operator $\mathcal{T}_{C}$, introduced at the beginning of Section 3.

The operator $\mathcal{T}_{C}$ maps an applied voltage $U$ at $\partial \Omega_{D}$ to the idealized data corresponding to the Neumann trace of the electric potential $\hat{V}$ at $\Gamma_{1} \subset \partial \Omega_{D}$, i.e.

$$
\begin{aligned}
\mathcal{T}_{C}: H^{3 / 2}\left(\partial \Omega_{D}\right) & \rightarrow H^{1 / 2}\left(\Gamma_{1}\right) \\
U & \left.\mapsto \frac{\partial \hat{V}}{\partial \nu}\right|_{\Gamma_{1}}
\end{aligned}
$$


where $\hat{V}$ solves:

$$
\left\{\begin{aligned}
\lambda^{2} \Delta \hat{V} & =\left(e^{V^{0}}+e^{-V^{0}}\right) \hat{V}+e^{V^{0}} \hat{u}+e^{-V^{0}} \hat{v} & & \text { in } \Omega \\
\hat{V} & =U & & \text { on } \partial \Omega_{D} \\
\nabla \hat{V} \cdot \nu & =0 & & \text { on } \partial \Omega_{N}
\end{aligned}\right.
$$

here $V^{0}$ is the solution the equilibrium case $(26)$ and $(\hat{u}, \hat{v})$ is the solution of the system

$$
\begin{cases}\operatorname{div}\left(\mu_{n} e^{V^{0}} \nabla \hat{u}\right)=Q_{0}\left(V^{0}, x\right)(\hat{u}+\hat{v}) & \text { in } \Omega \\ \operatorname{div}\left(\mu_{p} e^{-V^{0}} \nabla \hat{v}\right)=Q_{0}\left(V^{0}, x\right)(\hat{u}+\hat{v}) & \text { in } \Omega \\ \hat{u}=-U & \text { on } \partial \Omega_{D} \\ \hat{v}=U & \text { on } \partial \Omega_{D} \\ \frac{\partial \hat{u}}{\partial \nu}=\frac{\partial \hat{v}}{\partial \nu}=0 & \text { on } \partial \Omega_{N}\end{cases}
$$

Using a priori estimates of the solution of the Poisson equation, we conclude that $\hat{V}$ depends continuously on the boundary data as well as on the functions $\hat{u}$ and $\hat{v}$, appearing on the right hand side of the PDE. Further, we know that the map $U \mapsto(\hat{u}, \hat{v})$ is well-defined. Therefore, we can deduce the well-definedness of the application $\mathcal{T}_{C}$, for each doping profile $C$ in

$$
\left\{C \in L^{2}(\Omega) ; C_{m} \leq C(x) \leq C_{M} \text {, a.e. in } \Omega\right\} .
$$

The continuity of $\mathcal{T}_{C}$ can be proved in an analogous way. Furthermore, repeating the argumentation used for the operator $\Sigma^{\prime}(0)$, one can prove boundedness and compactness of the linear operator $\mathcal{T}_{C}$.

To obtain the abstract formulation of the inverse problem related to the operator $\mathcal{T}_{C}$, we take into account the more realistic case of a finite number of measurements:

1) Parameter: $C=C(x) \in L^{2}(\Omega)=: \mathcal{X}$;

2) Output: $Y=\left\{\mathcal{T}_{C}\left(U_{j}\right)\right\}_{j=1}^{N} \in\left[L^{2}\left(\Gamma_{1}\right)\right]^{N}=: \mathcal{Y}$;

3) Parameter-to-output map: $F: \mathcal{X} \rightarrow \mathcal{Y}$;

for fixed $U_{j} \in H^{3 / 2}\left(\partial \Omega_{D}\right)$ with $\left\|U_{j}\right\|$ small. The domain of definition of the operator $F$ is the same as in the case of the $\mathrm{V}-\mathrm{C}$ map. The parameter-tooutput operator is defined by

$$
\begin{aligned}
F: D(F) \subset \mathcal{X} & \rightarrow \mathcal{Y} \\
C & \mapsto\left\{\mathcal{T}_{C} U_{j}\right\}_{j=1}^{N}
\end{aligned}
$$

The well-definedness of the operator $F$ follows from the one of $\mathcal{T}_{C}$. The Fréchet-differentiability of the parameter-to-output operator can be proved analogously as in the case of full voltage-current data. 


\subsection{Laser-beam-inducted current measurements}

In this subsection we analyze the inverse problem related to the laser-beaminducted current (LBIC) image. This is a newly developed non-destructive optical technique for the detection of semiconductor properties. In this technique a laser beam is applied to the semiconductor body to induce currents to flow through the ohmic contacts on the boundary. The LBIC image consists of measurements of the local current $i(x)$ flowing out through one contact $\left(\Gamma_{1} \subset \partial \Omega_{D}\right)$ induced by a laser beam applied at location $x$ for all $x \in \Omega$. This image, considered as a mapping $\Omega \ni x \mapsto i(x) \in \mathcal{R}$, is considered to contain information about the doping profile. Therefore, the relation between the LBIC image and the doping profile can be modeled as an inverse problem for the system of drift-diffusion equations.

Let $\left(V^{0}, \hat{u}, \hat{v}\right)$ be the solution of system 26$)$, 32 . According to the representation formula (31), the LBIC image can be rewritten in the form

$$
i(x)=\tilde{v}(x)-\tilde{u}(x), x \in \Omega .
$$

The equilibrium potential $V^{0}$ satisfying $(26)$ is determined uniquely by the doping profile $C(x)$ and vice versa. Therefore, reconstructing the doping profile $C(x)$ from the LBIC image is equivalent to reconstructing the exponential of equilibrium potential $e^{V^{0}}$ from the representation $i(x)$ of the LBIC image.

In [23, 24] the uniqueness of the inverse problem is analyzed. In [24] a one dimensional problem is considered and the non-uniqueness of the inverse problem is proven. We shall next address this result.

A measurement $i(x)$ for $x \in \Omega$ is said to be attainable, if $i(x)=\hat{v}(x)-\hat{u}(x)$ with $(\hat{u}, \hat{v})$ being the solution of $(32)$ for some potential $V^{0}$. Let us for a moment consider the one dimensional version of system $(32)$ for $\Omega=(0,1)$

$$
\begin{cases}\left(\mu_{n} e^{V^{0}} \tilde{u}^{\prime}\right)^{\prime}=Q_{0}\left(V^{0}, x\right)(\tilde{u}-\tilde{v}) & x \in(0,1) \\ \left(\mu_{p} e^{-V^{0} \tilde{v}} \tilde{v}^{\prime}\right)^{\prime}=Q_{0}\left(V^{0}, x\right)(\tilde{v}-\tilde{u}) & x \in(0,1) \\ \tilde{u}(0)=\tilde{v}(0)=1 & \\ \tilde{u}(1)=\tilde{v}(1)=0 & \end{cases}
$$

In this case, the objective is to reconstruct $V^{0}$ (or alternatively $e^{V^{0}}$ ) from given $i(x)=\tilde{u}(x)-\tilde{v}(x)$. The next result establishes a necessary and sufficient condition for attainability of a measurement $i(x)$.

Proposition 5 [24, Theorem 2.1] A measurement $i(x)$ is attainable if and only if there exists constants $c_{1}$ and $c_{2}$ so that the equation

$$
\left(c_{1}-\frac{Q_{0}}{\mu_{n}} I(x)\right) Y(x)+i^{\prime}(x)-\left(c_{2}+\frac{Q_{0}}{\mu_{p}} I(x)\right) Y(x)^{-1}=0,
$$

has a positive solution $Y(x)$ for each $x \in(0,1)$, and $Y(x)$ satisfies the integral equation

$$
1+\int_{0}^{1}\left(c_{1}-\frac{Q_{0}}{\mu_{n}} I(x)\right) Y(x) d x=0 .
$$


Here $I(x)=\int_{0}^{x} i(\xi) d \xi$. Furthermore, if $i(x)$ is attainable then the constants $c_{1}$ and $c_{2}$ are nonpositive.

Sketch of the proof:

To prove the necessity, one integrates the differential equations in (35) and obtain a representation for $i^{\prime}(x)$. The attainability of $i(x)$ follows from the fact that $Y(x)=e^{-V^{0}(x)}$ satisfies both the quadratic equation and the integral equation of the proposition.

To prove the sufficiency, one sets

$$
\begin{aligned}
& \hat{u}(x)=1+\int_{0}^{x}\left(c_{1}-\frac{Q_{0}}{\mu_{n}} I(\xi)\right) Y(\xi) d \xi \\
& \hat{v}(x)=1+\int_{0}^{x}\left(c_{2}-\frac{Q_{0}}{\mu_{p}} I(\xi)\right) Y(\xi)^{-1} d \xi
\end{aligned}
$$

and obtain in a straightforward way that $(\hat{u}, \hat{v})$ solve $\sqrt{35}$ for $V^{0}(x)=-\ln Y(x)$. From an obvious substitution follows $\hat{v}-\hat{u}=\int_{0}^{x} i^{\prime}(\xi) d \xi$.

According to this result, the attainability of a measurement $i(x)$ is equivalent to the solvability of a quadratic equation for $Y(x)$. Notice that, if the constants $c_{1}$ and $c_{2}$ are known, then the potential is obtained simply by $V^{0}(x)=-\ln Y(x)$.

A first identifiability result is given in 23 . In this paper, the authors prove that $i(x) \equiv 0$ if and only if $V^{0}(x) \equiv c$, for some real constant $c$ (see Theorem 3.2 in the reference above). Therefore, in general there is no uniqueness for the inverse problem. Using Proposition 5, the same authors manage to extend this first non-uniqueness result for the one-dimensional case presented above, as follows

Proposition 6 [24, Theorem 2.3] Let $i(x) \in C_{0}^{1}[0,1]$ be an attainable measurement and $V^{0}$ be the corresponding potential. Moreover, assume that the constants $c_{1}$ and $c_{2}$ found by Proposition 5 with respect to $Y(x)=e^{-V^{0}(x)}$ satisfy

$$
c_{1}<\frac{Q_{0}}{\mu_{n}} I_{\min } \quad \text { and } \quad c_{2}<-\frac{Q_{0}}{\mu_{p}} I_{\max },
$$

where $I_{\min }$ and $I_{\max }$ are respectively the maximum and the minimum of $I(x)$ in $[0,1]$. Then there is a one-parameter family $\{V(x)\}$, containing $V^{0}$ and strictly monotone in the parameter, that produces the same measurement $i(x)=$ $\hat{v}(x)-\hat{u}(x)$ from system (35).

Proposition 6 characterizes the nonuniqueness of the one-dimensional inverse problem for the LBIC operator. Therefore, more information is needed to possibly recover $V^{0}(x)$ from $i(x)$ uniquely. In the LBIC technique it is reasonable to assume that doping profile is known on the boundary where the ohmic contacts are made $\left(\partial \Omega_{D} \subset \partial \Omega\right)$. Thus, if we assume that $V^{0}$ is given at $x=0$, we gain another constraint for $c_{1}$ and $c_{2}$, namely

$$
c_{1} e^{-V^{0}(0)}-i^{\prime}(0)-c_{2} e^{V^{0}(0)}=0 .
$$


This additional constraint ensures the unique recovery of $V^{0}(x)$ from the LBIC image $i(x)$ among the monotone one-parameter family $\{V(x)\}$ described in Proposition 6. Notice that this class of potentials does not include all possible solutions to the inverse problem. Therefore, the above constraint does not lead to uniqueness of solutions of the one-dimensional inverse problem in general.

In 24 the authors also propose an algorithm for the reconstruction of $V^{0}(x)$ from $i(x)$ based on Proposition 6 and the additional constraint (37). As discussed above, it is enough to reconstruct the constants $c_{1}$ and $c_{2}$. The proposed algorithm consists in a Gauss-Newton method for the minimization of a least square functional $J$ associated to the residual of the pairs $\left(c_{1}, c_{2}\right)$ in both 36 . and (37), namely

$$
J\left(c_{1}, c_{2}\right):=\frac{1}{2}\left(J_{1}\left(c_{1}, c_{2}\right)^{2}+J_{2}\left(c_{1}, c_{2}\right)^{2}\right),
$$

where

$$
\begin{aligned}
J_{1}\left(c_{1}, c_{2}\right) & :=\frac{1}{2} \int_{0}^{1} \sqrt{i^{\prime}(x)^{2}+4\left(c_{1}-\frac{Q_{0}}{\mu_{n}} I(\xi)\right)\left(c_{2}-\frac{Q_{0}}{\mu_{p}} I(\xi)\right)} d x-1 \\
J_{2}\left(c_{1}, c_{2}\right) & :=\left(c_{1} e^{-V^{0}(0)}-i^{\prime}(0)-c_{2} e^{V^{0}(0)}\right) e^{-\left|V^{0}(0)\right|} .
\end{aligned}
$$

In [25], a similar model based on the drift diffusion equations is used in order to analyze several parameter identification problems for semiconductor diodes by LBIC imaging. Numerical methods are developed for the simulation of the LBIC images of a diode as well as for the identification of parameters (junction depth, diffusion length equilibrium potential) from the LBIC image by least-squares formulation.

\subsection{Inverse doping profile: Identification}

In this subsection we consider the identification question related to the inverse doping profile problem, i.e. we shall focus on the following fundamental issue concerning the parameter identification problems:

Is the available data enough to determine uniquely the doping profile, or (alternatively) which set of data is sufficient to determine uniquely the doping profile?

In the one-dimensional case (i.e. $\Omega=(0, L))$ the identification problem was considered in [13. One can assume that the voltage is applied at $x=0$ and the measurements of both current and capacitance are taken at $x=L$. Therefore, a single measurement (reduced data) consists of two real numbers and full data, in this case, correspond to measure the current and/or the capacitance as a function of the applied voltage $U \in(-r, r)$, with appropriate $r \in \mathcal{R}$.

Arguing with the dimensionality of the parameter and data spaces as well as with structural properties of the operators $\Sigma_{C}$ and $\mathcal{T}_{C}$, the authors are able 
to fully analyze the one-dimensional inverse doping profile problem. The corresponding results are summarized in the following proposition.

Proposition 7 Let us consider the inverse doping profile for system (17)- 25 ) at the one-dimensional dimensional domain $\Omega=(0, L)$. The following assertion hold:

1. If one has access only to restricted data, even if it is possible to measure both, current and capacitance, the data are not sufficient to identify the doping profile;

2. If one has access to full data, it is not possible to uniquely identify the doping profile neither from current measurements nor from capacitance measurements.

One should notice that the doping profile $C=C(x)$ in this case is a function of a one-dimensional space variable.

In the same paper, the authors also consider the transient case of the onedimensional inverse doping profile problem. They prove, under special assumptions, that if both current and capacitance measurement are available, then the doping profile can be uniquely reconstructed from the data. Since we consider only the stationary drift-diffusion system in this paper, we shall not investigate this result here. For details, we refer to [13, Theorem 3].

In the one-dimensional case, the special problem in which the doping profile is a piecewise constant function of position is treated in 13. In this very particular case, the domain $\Omega$ can be split as $\bar{\Omega}=\bar{\Omega}_{n} \cup \bar{\Omega}_{p}$, such that $C(x) \equiv C_{+}$ in $\Omega_{n}$ and $C(x) \equiv C_{-}$in $\Omega_{p}$. This problem is also known as identification of $P-N$ junctions. The authors prove that reduced current data suffice to uniquely identify the exact location of the P-N junctions (i.e., $\bar{\Omega}_{n} \cap \bar{\Omega}_{p}$ ) if the number of junctions is lower or equal to two (see [13, Theorem 4]).

The two-dimensional case is considered in [12, 13. Particularly interesting is the inverse problem related to the $\mathrm{V}-\mathrm{C}$ map for the linearized unipolar case close to equilibrium (see Subsection 2.4), which can be directly related to the inverse problem in electrical impedance tomography.

The inverse problem in the unipolar case corresponds to the determination of the doping profile $C$ from the map

$$
\begin{aligned}
\Sigma_{C}^{\prime}(0): H^{3 / 2}\left(\partial \Omega_{D}\right) & \rightarrow H^{1 / 2}\left(\Gamma_{1}\right) \\
U & \left.\mapsto\left(\hat{J}_{n} \cdot \nu\right)\right|_{\Gamma_{1}}
\end{aligned}
$$

where $\left(u, V^{0}\right)$ is the solution of the system in 28$)$.

As already observed in Subsection 2.4 it follows from the fact that $\left.V^{0}\right|_{\partial \Omega_{D}}=$ $V_{\text {bi }}$ is a known function, that the current data $\left.J_{n} \cdot \nu\right|_{\Gamma_{1}}$ can be directly substituted by the Neumann data $\left.u_{\nu}\right|_{\Gamma_{1}}$. Therefore, the inverse problem can be divided in 2 steps: 
1) Define $\gamma:=e^{V^{0}}$ and identify $\gamma$ in

$$
\begin{cases}\operatorname{div}(\gamma \nabla u)=0 & \text { in } \Omega \\ u=U & \text { on } \partial \Omega_{D} \\ u_{\nu}=0 & \text { on } \partial \Omega_{N}\end{cases}
$$

from the Dirichlet-to-Neumann map: $\left.\left.u\right|_{\partial \Omega_{D}} \mapsto u_{\nu}\right|_{\Gamma_{1}}$;

2) Obtain the doping profile $C(x)$ from: $C=\gamma-\lambda^{2} \Delta(\ln \gamma)$.

The identification problem in 1) corresponds to the electrical impedance tomography (or inverse conductivity problem) in elliptic equations with mixed boundary data. For the case of the full Dirichlet-to-Neumann operator, i.e. $\Gamma_{1}=\partial \Omega_{D}=\partial \Omega$, this inverse problem has been intensively analyzed in the literature over the last fifteen years. Using different regularity assumptions on the conductivity $\gamma$, many authors proved that the coefficient $\gamma(x)$ of the elliptic equation $\nabla \cdot(\gamma \nabla u)=0$ is uniquely determined by the corresponding Dirichletto-Neumann map on the boundary (a historical overview can be found in [9]).

In the sequel we mention a result due to A. Nachman for two-dimensional domains. The proof of this theorem gives a constructive procedure for recovering $\gamma$ from the Dirichlet-to-Neumann map.

Proposition 8 [47, Theorem 1] Let $\Omega$ be bounded and Lipschitz. Further, let $\gamma_{i} \in L^{\infty}(\Omega) \cap W^{2, p}(\Omega), i=1,2$, for some $p>1$ with positive lower bound. Then, the equality of the Dirichlet-to-Neumann maps

$$
\begin{aligned}
\Lambda_{i}: H^{1 / 2}(\partial \Omega) & \rightarrow H^{-1 / 2}(\partial \Omega) \\
u & \mapsto u_{\nu}
\end{aligned}
$$

for the solutions of $\operatorname{div}\left(\gamma_{i} \nabla u\right)=0$, implies $\gamma_{1}=\gamma_{2}$.

According to Proposition $3, H^{2}$-regularity of the solution $(V, u, v)$ of system 17) - 25) can be obtained under stronger regularity assumptions on both the mixed boundary conditions, and the domain. Using this regularity result, it is possible to adapt Proposition 8 for the identification problem in the unipolar case for the operator $\Sigma_{C}^{\prime}(0): H^{3 / 2}\left(\partial \Omega_{D}\right) \rightarrow H^{1 / 2}\left(\Gamma_{1}\right)$ in the idealized case $\Gamma_{1}=\partial \Omega_{D}=\partial \Omega$, as follows

Proposition 9 [12, Theorem 4.2] Let $\Omega \subset \mathbb{R}^{2}$ be bounded and Lipschitz. Further, let $\Gamma_{1}=\partial \Omega_{D}=\partial \Omega$. Then, given two doping profiles $C_{1}, C_{2} \in D(F)$, the equality $\Sigma_{C_{1}}^{\prime}(0)=\Sigma_{C_{2}}^{\prime}(0)$ implies $C_{1}=C_{2}$.

If we consider the solution of (17)-25) to be only in $H^{1}$ (see Proposition 1), we can alternatively consider the following identifiability result from Brown and Uhlmann for $W^{1, p}(\Omega), p>2$, conductivities: 
Proposition 10 [11, Theorem 4.1] Let $\Omega$ be bounded and Lipschitz. Further, let $\gamma_{1}$ and $\gamma_{2}$ be two conductivities with $\nabla \gamma_{i}$ in $L^{p}(\Omega), p>2$. Then, the equality of the Dirichlet-to-Neumann maps

$$
\begin{aligned}
\Lambda_{i}: H^{1 / 2}(\partial \Omega) & \rightarrow H^{-1 / 2}(\partial \Omega) \\
u & \mapsto u_{\nu}
\end{aligned}
$$

for the solutions of $\operatorname{div}\left(\gamma_{i} \nabla u\right)=0$, implies $\gamma_{1}=\gamma_{2}$.

Using this identifiability result, it is possible to deduce, for the operator $\Sigma_{C}^{\prime}(0): H^{1 / 2}\left(\partial \Omega_{D}\right) \rightarrow H^{-1 / 2}\left(\Gamma_{1}\right)$ an analog result to the one presented in Proposition 9. Notice that this result is particularly interesting for the case of zero space charge (see Subsection 3.1 for the $\mathrm{V}-\mathrm{C}$ map, allowing to prove identifiability of doping profiles $C \in L^{\infty}(\Omega) \cap W^{1, p}(\Omega)$.

\section{Numerical experiments}

In this section we derive a numerical method to identify the doping profile in the linearized unipolar case close to equilibrium (28). In this particular case, due to the assumptions $p \equiv 0$ and $Q \equiv 0$, the Poisson equation and the continuity equation for the electron density $n$ decouple, and we have to identify $C=C(x)$ in

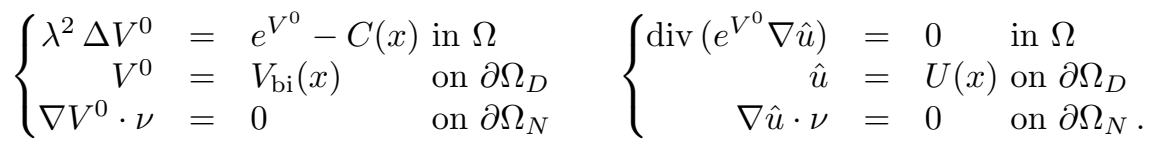

Notice that, due to the relation $\hat{J} \cdot \nu=\mu_{n} e^{V^{0}} \hat{u}$, the Neumann boundary condition $\left.\hat{J}_{n} \cdot \nu\right|_{\partial \Omega_{N}}=0$ in 28 can be substituted by $\nabla \hat{u} \cdot \nu=0$ on $\partial \Omega_{N}$. As already observed in Subsection 3.4 , we can write $\gamma(x):=e^{V^{0}(x)}$; solve the parameter identification problem

$$
\left\{\begin{aligned}
\operatorname{div}(\gamma \nabla \hat{u}) & =0 & & \text { in } \Omega \\
\hat{u} & =U(x) & & \text { on } \Omega_{D} \\
\nabla \hat{u} \cdot \nu & =0 & & \text { on } \Omega_{N}
\end{aligned}\right.
$$

for the function $\gamma$; and finally evaluate

$$
C(x)=\gamma-\lambda^{2} \Delta(\ln \gamma) .
$$

Since the evaluation of $C$ from $\gamma$ can be explicitely performed (a direct problem) and is a well posed procedure, we shall focus on the problem of identifying the function parameter $\gamma$ in (39). Therefore, the inverse problem of identifying the doping profile $C(x)$ in the linearized unipolar model 28) corresponds to the identification of $\gamma(x)$ in $(39)$ from the Dirichlet to Neumann (DtN) map

$$
\begin{aligned}
\Lambda_{\gamma}: H^{3 / 2}\left(\partial \Omega_{D}\right) & \rightarrow H^{1 / 2}\left(\Gamma_{1}\right) \\
U & \left.\mapsto \gamma \frac{\partial u}{\partial \nu}\right|_{\Gamma_{1}}
\end{aligned}
$$


As we saw in Subsection 3.4 the DtN operator is given by $\Lambda_{\gamma}=\Sigma_{C}^{\prime}(0)$.

Notice that, due to the nature of the physical problem related to the driftdiffusion equations, we can consider as inputs for the DtN map only functions of the type:

$$
U=\left\{\begin{array}{cl}
\tilde{U}, & \text { at } \partial \Omega_{D} \backslash \Gamma_{1} \\
0, & \text { at } \Gamma_{1}
\end{array} .\right.
$$

Furthermore, the outputs or measurements are only available at $\Gamma_{1}$. This is the basic difference between the parameter identification problem in 390 and the inverse problem in electrical impedance tomography, i.e. the fact that both Dirichlet (input) and Neumann (output) are prescribed only at specific parts of the boundary. For this special inverse problem (with mixed boundary data) there are so far no analytical results concerning identifiability and the few numerical results in the literature are those discussed in [12, 13, 25].

We shall work with a reduced set of data, as described in Subsection 3.1, i.e. within the following framework:

1) Parameter: $\gamma=\gamma(x) \in H^{2}(\Omega)=: \mathcal{X}$;

2) Input (fixed): $U_{j} \in H^{1 / 2}\left(\partial \Omega_{D}\right),\left.U_{j}\right|_{\Gamma_{1}}=0,1 \leq j \leq N$;

3) Output (data): $Y=\left\{\left.\gamma \frac{\partial \hat{u}_{j}}{\partial \nu}\right|_{\Gamma_{1}}\right\}_{j=1}^{N} \in\left[L^{2}\left(\Gamma_{1}\right)\right]^{N}=: \mathcal{Y}$;

(here $u_{j}$ is the solution of 39 ) for $U=U_{j}$ )

4) Parameter-to-output map: $F: \mathcal{X} \rightarrow \mathcal{Y}$.

The domain of definition of the operator $F$ is

$$
D(F):=\left\{\gamma \in H^{2}(\Omega) ; \gamma(x) \geq \gamma_{-}>0, \text { in } \Omega\right\},
$$

where $\gamma_{-}$is an appropriate positive constant. We shall denote the noisy data by $Y^{\delta}$ and assume that the data error is bounded by

$$
\left\|Y-Y^{\delta}\right\| \leq \delta .
$$

Thus, we are able to represent the inverse doping problem in the abstract form

$$
F(\gamma)=Y^{\delta}
$$

A common technique to solve the inverse problem in (40) is the output leastsquare family of methods. Basically, all output least-square methods minimize iteratively the residual functional related to 40 with some Newton-type method [1, 2, 3, 4, 5, 16, 19, 29, 33, 36, 37, 51. In the literature, one can find several applications of such methods for the electrical impedance tomography problem (see, e.g., 10, 17, 18, 157).

A simple and robust iterative method to solve the problem in $(40)$ is the so called Landweber iteration [16, 19, 21, 31, in which the k-step is described by

$$
\gamma_{k+1}^{\delta}=\gamma_{k}^{\delta}-F^{\prime}\left(\gamma_{k}^{\delta}\right)^{*}\left(F\left(\gamma_{k}^{\delta}\right)-Y^{\delta}\right) .
$$


This iteration is known to generate a regularization method for the inverse problem, the stopping index playing the rule of the regularization parameter (for regularization methods see, e.g., [19, 20, 21, 46, 55]).

For our numerical experiments, we propose an iterative method of adjoint type in order to solve the identification problem (39), the so called LandweberKaczmarz method. This method derives from the coupling of the strategies of the Landweber iteration and the Kaczmarz method. The Kaczmarz method is a fixed point algorithm which has been proven to be efficient for solving inverse problems in Tomography [7, 8, 27, 39, 48. For a detailed analysis of the method we refer to [7, 45] for the finite dimensional setting and to [6, 43, 44, for the infinite dimensional setting.

A detailed analysis of the Landweber-Kaczmarz method can be found in [40. It is worth mentioning that this method has already been successfully applied to the electrical impedance tomography problem [47. To formulate the method, we need first define the parameter-to-output maps

4') Operators $\mathcal{F}_{j}$ for $j=1, \ldots, N$ :

$$
\begin{aligned}
\mathcal{F}_{j}: H^{2}(\Omega) & \rightarrow L^{2}\left(\Gamma_{1}\right) \\
\gamma & \left.\mapsto \gamma \frac{\partial \hat{u}_{j}}{\partial \nu}\right|_{\Gamma_{1}}
\end{aligned}
$$

Now, setting $Y_{j}:=\mathcal{F}_{j}(\gamma)$ for $1 \leq j \leq N$, the Landweber-Kaczmarz iteration can be written as:

$$
\gamma_{k+1}^{\delta}=\gamma_{k}^{\delta}-\mathcal{F}_{k}^{\prime}\left(\gamma_{k}^{\delta}\right)^{*}\left(\mathcal{F}_{k}\left(\gamma_{k}^{\delta}\right)-Y_{k}^{\delta}\right),
$$

for $k=1,2, \ldots$, where we adopted the notation

$$
\mathcal{F}_{k}:=\mathcal{F}_{j}, \quad Y_{k}^{\delta}:=Y_{j}^{\delta}, \quad \text { with } k=i \cdot N+j, \quad \text { and }\left\{\begin{array}{l}
i=0,1, \ldots \\
j=1, \ldots, N
\end{array} .\right.
$$

Each step of the Landweber-Kaczmarz method consists in one step of the Landweber iteration with respect to the $j$-th component of the residual $F(\gamma)-Y$. These steps are performed in a cyclic way for each one of the residual components $\mathcal{F}_{j}(\gamma)-Y_{j}, j=1, \cdots, N$.

As far as the implementation of the method is concerned, it is enough to describe the general step of the Landweber iteration. The variational formulation of the iterative step in 41 reads

$$
\left\langle\gamma_{k+1}-\gamma_{k}, h\right\rangle_{L^{2}(\Omega)}=-\left\langle\mathcal{F}_{k}^{\prime}\left(\gamma_{k}\right) h, \mathcal{F}_{k}\left(\gamma_{k}\right)-Y_{k}\right\rangle_{L^{2}(\Omega)},
$$

where $h \in H^{1}(\Omega)$ is a test function (to simplify the notation we set $\delta=0$, i.e. $Y_{k}^{\delta}=Y_{k}$ and $\left.\gamma_{k}^{\delta}=\gamma_{k}\right)$.

In order to compute the inner product on the right hand side of 42 , we use the identity:

$$
\left\langle\mathcal{F}^{\prime}(\gamma) h, z\right\rangle_{L^{2}\left(\Gamma_{1}\right)}=\int_{\Omega} h \nabla G(\gamma) \cdot \nabla \Phi(\gamma) d x
$$


for $z \in L^{2}\left(\Gamma_{1}\right)$, where the $H^{1}(\Omega)$-function $\Phi(a)$ solves

$$
\left\{\begin{array}{rlrl}
-\nabla(a(x) \nabla w) & =0, & & \text { in } \Omega \\
w=z, & & \text { on } \Gamma_{1} \\
w=0, & & \text { on } \partial \Omega / \Gamma_{1}
\end{array}\right.
$$

and the $H^{1}(\Omega)$-function $G(a)$ solves

$$
\left\{\begin{array}{rlrl}
-\nabla(a(x) \nabla w) & =0, & & \text { in } \Omega \\
a(x) w_{\nu}=0, & & \text { on } \partial \Omega_{N} \\
w & =g, & & \text { on } \partial \Omega_{D}
\end{array}\right.
$$

Indeed, since the Fréchet derivative of the operator

$$
\begin{aligned}
\Psi: H^{2}(\Omega) & \rightarrow H^{1 / 2}(\partial \Omega) \\
a & \left.\mapsto a w_{\nu}\right|_{\partial \Omega}
\end{aligned} \quad \text { where } \quad\left\{\begin{aligned}
-\nabla(a(x) \nabla w)=f, & & \text { in } \Omega \\
w=g, & & \text { on } \partial \Omega
\end{aligned}\right.
$$

in the direction $h \in H^{2}(\Omega)$ is given by

$$
\Psi^{\prime}(a) \cdot h=\left(h G_{\nu}(a)+a \psi_{\nu}\right),
$$

where

$$
\left\{\begin{aligned}
-\nabla(a(x) \nabla \psi) & =\nabla(h(x) \nabla G(a)), & & \text { in } \Omega \\
\psi & =0, & & \text { on } \partial \Omega
\end{aligned}\right.
$$

we have

$$
\begin{aligned}
&\left\langle\mathcal{F}^{\prime}(\gamma) h, z\right\rangle_{L^{2}\left(\Gamma_{1}\right)}=\int_{\Gamma_{1}} z\left(h(G(\gamma))_{\nu}+\gamma \psi_{\nu}\right) \\
&=\int_{\Gamma_{1}} z h(G(\gamma))_{\nu}+\int_{\Gamma_{1}} \Phi(\gamma) \gamma \psi_{\nu}+\int_{\partial \Omega_{D} / \Gamma_{1}} \Phi(\gamma) \gamma \psi_{\nu}+\int_{\partial \Omega_{N}} \Phi(\gamma) \gamma \psi_{\nu} \\
&=\int_{\Gamma_{1}} z h(G(\gamma))_{\nu}+\int_{\Omega} \nabla(\gamma \nabla \psi) \Phi(\gamma)+\int_{\Omega} \gamma \nabla \psi \cdot \nabla \Phi(\gamma) \\
&=\int_{\Gamma_{1}} z h(G(\gamma))_{\nu}-\int_{\Omega} \nabla(h \nabla G(\gamma)) \Phi(\gamma)+\int_{\partial \Omega} \psi\left(\gamma(\Phi(\gamma))_{\nu}\right) \\
&-\int_{\Omega} \psi \nabla(\gamma \nabla \Phi(\gamma)) \\
&= \int_{\Gamma_{1}} z h(G(\gamma))_{\nu}-\left[\int_{\Gamma_{1}} h(G(\gamma))_{\nu} \Phi(\gamma)+\int_{\partial \Omega / \Gamma_{1}} h(G(\gamma))_{\nu} \Phi(\gamma)\right] \\
&+\int_{\Omega} h \nabla G(\gamma) \cdot \nabla \Phi(\gamma)
\end{aligned}
$$

and 43 follows. Therefore, the term on the right hand side of 42 can be evaluated by using formula 43 with $z=\mathcal{F}_{k}\left(\gamma_{k}\right)-Y_{k}$. 


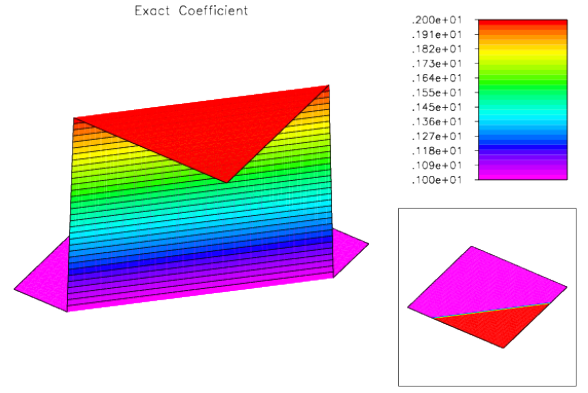

(a)

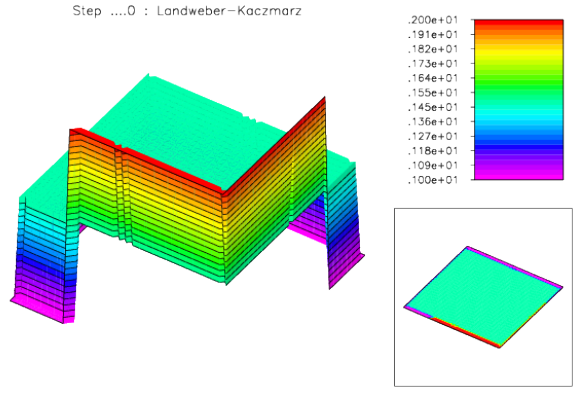

(b)

Figure 2: Picture (a) shows the exact coefficient $\bar{\gamma}(x)$ to be reconstructed. On picture (b), the initial condition for the Landweber-Kaczmarz iteration is shown.

For the concrete numerical test performed in this paper, $\Omega \subset \mathcal{R}^{2}$ is the unit square, and the boundary parts are defined as follows

$$
\begin{gathered}
\Gamma_{1}:=\left\{(x, 1) ; x \in\left(0, \frac{1}{2}\right)\right\}, \quad \partial \Omega_{D}:=\Gamma_{1} \cup\{(x, 0) ; x \in(0,1)\} \\
\partial \Omega_{N}:=\{(0, y) ; y \in(0,1)\} \cup\{(1, y) ; y \in(0,1)\} \cup\left\{(x, 1) ; x \in\left(\frac{1}{2}, 1\right)\right\} .
\end{gathered}
$$

The fixed inputs $U_{j} \in H^{1 / 2}\left(\partial \Omega_{D}\right)$, are chosen to be piecewise linear functions supported in $\partial \Omega_{D} / \Gamma_{1}$

$$
U_{j}(x):=\left\{\begin{aligned}
1-\frac{1}{h}\left|x-x_{j}\right|, & \left|x-x_{j}\right| \leq h \\
0, & \text { else }
\end{aligned}\right.
$$

where the points $x_{j}$ are equally spaced in the interval $(0,1)$. The doping profile $C=C(x)$ to be reconstructed corresponds to the function $\bar{\gamma}(x)$ shown in Figure 2 (a). In this figure, as well as in the forthcoming ones, $\Gamma_{1}$ appears in the lower right part of the picture and $\partial \Omega_{D} / \Gamma_{1}$ appears on the top (the origin corresponds to the upper right corner).

To generate the problem data, one has to solve the direct problem in 39 for each input function $U_{j}, j=1, \cdots, N$. In order to avoid the so called inverse crimes, these problems are solved using adaptive mesh regularization and a piecewise linear finite element base with approximately 8000 nodal points. This mesh is different from the one used to solve the mixed elliptic boundary value problems, related to the implementation of the Landweber-Kaczmarz method. These problems are solved using a multigrid finite element method at uniformly refined grids with approximately 2000 nodal points.

We still have to take into account an important issue concerning the stability of the numerical implementation. Due to the particular geometry of $\Omega$ (note that $\partial \Omega_{D}$ and $\partial \Omega_{N}$ meet at angles of $\pi$ and $\pi / 2$ ), both the solution of the direct elliptic (mixed) problems as well as the solution of the boundary value problems involved in the implementation of the Landweber-Kaczmarz method are not in $H^{2}(\Omega)$ (see remark at the end of Subsection 2.2p. 


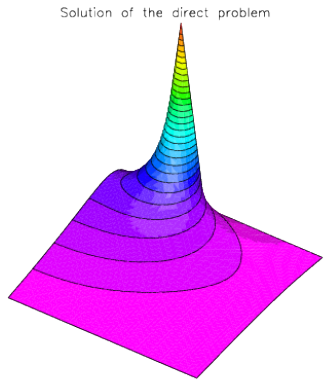

(a)

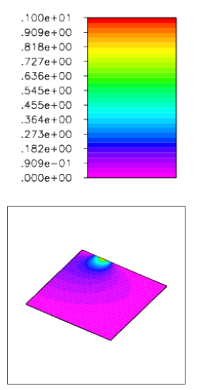

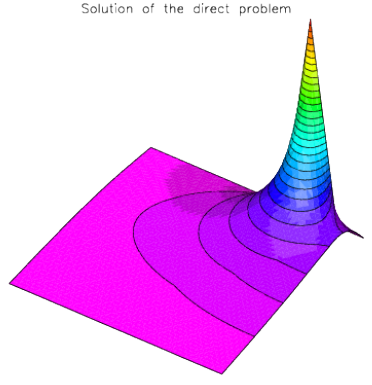

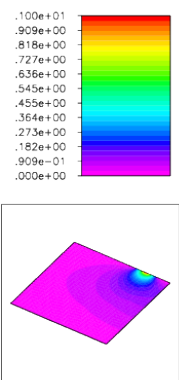

(b)

Figure 3: Pictures (a) and (b) show different pairs of (Dirichlet,Neumann) data used in separate runs of the Landweber-Kaczmarz iteration.

Because of this lack of regularity in the solution of the elliptic boundary value problems, the numeric implementation of the Landweber-Kaczmarz method has shown to be very unstable. After a few iterative steps the sequence $\gamma_{k}$ became unbounded, the main singularity appearing near the boundary (note that we assume $C$, or equivalently $\gamma$, to be known at the boundary) close to the contact points between $\partial \Omega_{D}$ and $\partial \Omega_{N}$. This phenomena could be observed even if we started the iteration with $\gamma_{0}(x)=\gamma^{\dagger}(x)$, the exact solution of the inverse problem.

In order to avoid the instability described above, we make the additional assumption that the doping profile is known in a thin strip close to $\partial \Omega$. Therefore, we only have to reconstruct the values of $\gamma(x)$ at a subdomain $\tilde{\Omega} \subset \subset \Omega$. With this extra assumption, the numerical implementation becomes stable and we are able to characterize (numerically) the exact solution $\gamma^{\dagger}(x)$ as a fixed point of the Landweber-Kaczmarz iteration. It is worth mentioning that this sort of assumption is very common in the literature (see, e.g., [9] and the references therein) and has been used since the early investigations of the electrical impedance tomography, in order to insure extra regularity for both numerical and analytical approaches (see [52]).

In Figure 2(b) the initial condition for the Landweber-Kaczmarz method is shown. Comparing the initial condition with the exact solution, one can observe that the values of $\gamma_{0}(x)$ and $\gamma^{\dagger}(x)$ coincide close to $\partial \Omega$. This is in accordance with the assumption above. Close to the boundary $\partial \Omega$, the values of $\gamma_{k}$ are not iterated, and we actually have $\gamma_{k}=\gamma^{\dagger}$ at $\Omega / \tilde{\Omega}$.

Concerning the amount of information used in the reconstruction, we implemented (for comparison purposes) the Landweber-Kaczmarz iteration in the case where a single pair of Dirichlet and Neumann data was available. In this case, the Landweber-Kaczmarz method reduces to the Landweber iterative method. This experiment is interesting, since it shows that the quality of the reconstruction is better at the part of the domain $\Omega$ which is closer to the support of the applied voltage. In Figure 4 we present the evolution of the 
Landweber iteration for $N=1$ and

$$
U_{1}(x):=\left\{\begin{aligned}
1-8\left|x-\frac{6}{8}\right|, & \left|x-\frac{6}{8}\right| \leq \frac{1}{8} \\
0, & \text { else }
\end{aligned}\right.
$$

The solution of the direct problem corresponding to this choice of $U_{1}$ is shown in Figure 3 (a). In Figure 5 we present the evolution of the Landweber iteration for $N=1$ and

$$
U_{1}(x):=\left\{\begin{aligned}
1-8\left|x-\frac{2}{8}\right|, & \left|x-\frac{2}{8}\right| \leq \frac{1}{8} \\
0, & \text { else }
\end{aligned}\right.
$$

The solution of the direct problem corresponding to this choice of $U_{1}$ is shown in Figure 3 (b).

In Figure 6 we present the reconstruction results obtained by the LandweberKaczmarz iteration for $N=9$, i.e. nine pair of Dirichlet and Neumann Data. We implemented the method with different amounts of data (i.e. different values of $N)$. For $N \geq 5$ the numerical results were very close. The results correspond to exact data, i.e. no noise was introduced. The numerics have shown to be sensible with respect to noise. Even though, we were able to obtain some acceptable results for noisy data. In Figure 7 we present the results obtained with a noise level of $10 \%$ (white noise).

\section{Acknowledgment}

M.B. and H.E. acknowledge financial support from the Austrian National Science Foundation FWF through project SFB F 013/08. A.L. is on leave from Department of Mathematics, Federal University of St. Catarina, Brazil; his work is supported by the Austrian Academy of Sciences and CNPq, grant 305823/20035. P.M. acknowledges support from the Austrian National Science Foundation FWF through his Wittgenstein Award.

\section{References}

[1] BAKUshinskIĬ, A.B. On a convergence problem of the iterative-regularized Gauss-Newton method, Comput. Math. Phys. 32 (1993), 1353-1359

[2] BakushinskiI , A.B. Iterative methods for solving nonlinear operator equations without regularity. A new approach, Russian Acad. Sci. Dokl. Math. 47 (1993), 451-454

[3] BAKUshinskiĬ, A.B. Universal linear approximations of solutions to nonlinear operator equations and their application, J. Inverse Ill-Posed Probl. 5 (1997), 507-521

[4] Bakushinskĭ, A.B.; Goncharskil̆, A.V. Iterative methods for solving ill-posed problems, Nauka, Moscow, 1989 (in Russian) 
[5] BakushinskiĬ, A.B.; GoncharskiĬ, A.V. Ill-posed Problems: Theory and Applications, Kluwer, Dordrecht, 1994

[6] BAnK, R.E.; Rose, D.J. Analysis of a multilevel iterative method for nonlinear finite element equations, Math. Comp. 39 (1982), 453-465

[7] Baumeister, J. Stable Solution of Inverse Problems, Vieweg, Braunschweig, 1987

[8] Baumeister, J.; Scondo, W. Adaptive methods for parameter identification, in Optimization in mathematical physics (Oberwolfach, 1985), 87-116, Methoden Verfahren Math. Phys., 34, Lang, Frankfurt am Main, 1987

[9] BorceA, L. Electrical impedance tomography, Inverse Problems 18 (2002), R99-R136

[10] BorceA, L. A nonlinear multigrid for imaging electrical conductivity and permittivity at low frequency,. Inverse Problems 17 (2001), 329-359

[11] Brown, R.M.; Uhlmann, G.A. Uniqueness in the inverse conductivity problem for nonsmooth conductivities in two dimensions, Comm. Partial Differential Equations 22 (1997), 1009-1027

[12] Burger, M.; Engl, H.W.; Markowich, P.A.; Pietra, P. Identification of doping profiles in semiconductor devices, Inverse Problems $\mathbf{1 7}$ (2001), 1765-1795

[13] Burger, M.; Engl, H.W.; Markowich, P. Inverse doping problems for semiconductor devices, in: T.F.Chan et al, eds., Recent Progress in Computational and Applied PDEs (Kluwer Academic/Plenum Publishers, $2002,27-38$

[14] Burger, M.; Pinnau, R. Fast optimal design of semiconductor devices, SIAM J. Appl. Math. 64(2003), 108-126

[15] Busenberg, S.; Fang, W. Identification of semiconductor contact resistivity, Quart. Appl. Math. 49 (1991), 639-649

[16] Deuflhard, P.; Engl, H.W.; Scherzer, O. A convergence analysis of iterative methods for the solution of nonlinear ill-posed problems under affinely invariant conditions, Inverse Problems 14 (1998), 1081-1106

[17] Dines, K.A.; Lytle, R.J. Analysis of electrical conductivity imaging, Geophysics 46 (1981), 1025-1036

[18] Dobson, D.C. Convergence of a reconstruction method for the inverse conductivity problem, SIAM J. Appl. Math. 52 (1992), 442-458

[19] Engl, H.W.; Hanke, M.; Neubauer, A. Regularization of Inverse Problems, Kluwer Academic Publishers, Dordrecht, 1996 (Paperback: 2000) 
[20] Engl, H.W.; Kunisch, K.; Neubauer, A. Convergence rates for Tikhonov regularization of nonlinear ill-posed problems, Inverse Problems 5 (1989), 523-540

[21] Engl, H.W.; Scherzer, O. Convergence rates results for iterative methods for solving nonlinear ill-posed problems, in D. Colton et al eds., Surveys on solution methods for inverse problems, 7-34, Springer, Vienna, 2000

[22] Fang, W.; Cumberbatch, E. Inverse problems for metal oxide semiconductor field-effect transistor contact resistivity, SIAM J. Appl. Math. 52 (1992), 699-709

[23] FAnG, W.; Ito, K. Identifiability of semiconductor defects from LBIC images, SIAM J. Appl. Math. 52 (1992), 1611-1626

[24] FAng, W.; Ito, K. Reconstruction of semiconductor doping profile from laser-beam-induced current image, SIAM J. Appl. Math. 54 (1994), 10671082

[25] Fang, W.; Ito, K.; Redfern, D.A. Parameter identification for semiconductor diodes by LBIC imaging, SIAM J. Appl. Math. 62 (2002), 21492174

[26] P.Grisvard, Singularities in Boundary Value Problems, Springer-Verlag, Berlin, 1992

[27] Groetsch, C.W. Inverse Problems in the Mathematical Sciences, Vieweg, Braunschweig, 1993

[28] Gilbarg, D.; Trudinger, N. Elliptic Partial Differential Equations of Second Order, Springer-Verlag, New York, 1977

[29] Hanke, M. A regularizing Levenberg-Marquardt scheme, with applications to inverse groundwater filtration problems, Inverse Problems 13 (1997), $79-95$

[30] Hanke, M. Regularizing properties of a truncated Newton-CG algorithm for nonlinear inverse problems, Numer. Funct. Anal. Optim. 18 (1997), 971-993

[31] Hanke, M.; Neubauer, A.; Scherzer, O. A convergence analysis of the Landweber iteration for nonlinear ill-posed problems, Numer. Math. $\mathbf{7 2}$ (1995), 21-37

[32] Hinze, M.; Pinnau, R. An optimal control approach to semiconductor design, Math. Mod. Meth. Appl. Sci. 12 (2002), 89-107

[33] Hohage, T. Logarithmic convergence rates of the iteratively regularized Gauss-Newton method for an inverse potential and an inverse scattering problem, Inverse Problems 13 (1997), 1279-1299 
[34] Hohage, T. Convergence rates of a regularized Newton method in soundhard inverse scattering, SIAM J. Numer. Anal. 36 (1999), 125-142

[35] Isakov, V. Inverse problems for partial differential equations, Applied Mathematical Sciences, Springer-Verlag, New York, 1998

[36] Kaltenbacher, B. Some Newton-type methods for the regularization of nonlinear ill-posed problems, Inverse Problems 13 (1997), 729-753

[37] Kaltenbacher, B. On Broyden's method for the regularization of nonlinear ill-posed problems, Numer. Funct. Anal. Optim. 19 (1998), 807-833

[38] Khalil, N.; Faricelli, J.; Bell, D.; Selberherr, S. The extraction of two-dimensional MOS transistor doping via inverse modeling, IEEE Electron Device Lett. 16 (1995), 17-19

[39] Kirsch, A. An introduction to the mathematical theory of inverse problems, Springer-Verlag, New York, 1996

[40] Kowar, R.; Scherzer, O. Convergence analysis of a LandweberKaczmarz method for solving nonlinear ill-posed problems, in S.I. Kabanikhin et al eds., Ill-Posed and Inverse Problems, 253-270, VSP, Boston, 2002

[41] Markowich, P.A. The Stationary Semiconductor Device Equations, Springer-Verlag, Vienna, New York, 1986

[42] Markowich, P.A.; Ringhofer, C.A.; Schmeiser, C. Semiconductor Equations, Springer-Verlag, Vienna, New York, 1990

[43] McCormick, S.F. An iterative procedure for the solution of constrained nonlinear equations with application to optimization problems, Numer. Math. 23 (1975), 371-385

[44] McCormick, S.F. The methods of Kaczmarz and row orthogonalization for solving linear equations and least squares problems in Hilbert space, Indiana Univ. Math. J. 26 (1977), 1137-1150

[45] Meyn, K.H. Solution of underdetermined nonlinear equations by stationary iteration methods, Numer. Math. 42 (1983), 161-172

[46] Morozov, V.A. Methods for solving incorrectly posed problems, SpringerVerlag, New York, 1984

[47] Nachman, A.I. Global uniqueness for a two-dimensional inverse boundary value problem, Ann. of Math. 143 (1996), 71-96

[48] NatTerer, F. The mathematics of Computerized Tomography, Teubner/Wiley, Stuttgart, 1986 (Reprinted on SIAM Classics in Applied mathematics, 1991) 
[49] NATterer, F. Numerical solution of bilinear inverse problems, Technical Report 19/96-N, Fachbereich Mathematik der Universität Münster, 1996

[50] Natterer, F.; Wübbeling, F. Mathematical Methods in Image Reconstruction, SIAM, Philadelphia, 2001

[51] Scherzer, O. A posteriori error estimates for the solution of nonlinear ill-posed operator equations, Nonlinear Anal. 45 (2001), 459-481

[52] SCHERZER, O. Tikhonov regularization of nonlinear ill-posed problems with applications to parameter identification in partial differential equations, Dissertationen der Johannes-Kepler-Universität Linz, 86. Verband der Wissenschaftlichen Gesellschaften Österreichs, Vienna, 1991.

[53] Selberherr, S. Analysis and Simulation of Semiconductor Devices, Springer-Verlag, Vienna, New York, 1984

[54] Stockinger, M. Optimization ofultra-low-power CMOS transistors, $\mathrm{PhD}$ Thesis, University Vienna, 2000

[55] Tikhonov, A.N.; Arsenin, V.Y. Solutions of Ill-posed Problems, John Wiley \& Sons, New York, 1977

[56] Van Roosbroeck, W.R. Theory of flow of electrons and holes in germanium and other semiconductors, Bell Syst. Tech. J. 29 (1950), 560-607

[57] Yorkey, T.J.; Webster, J.G.; Tompkins, W.J. Compasring reconstruction algorythms for electrical impedance tomography, IEEE Trans. Biomed. Eng. 34 (1987), 843-852 

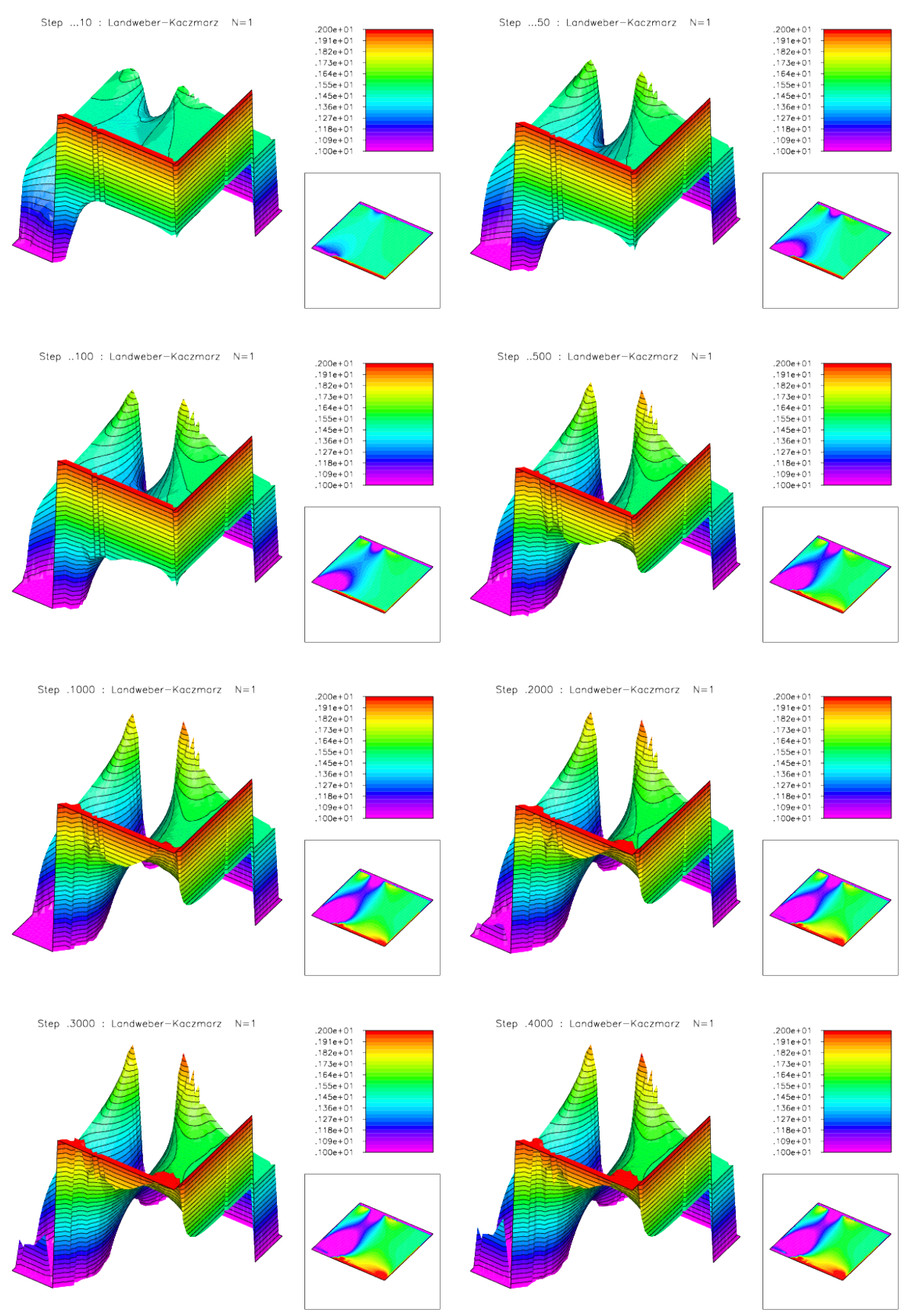

Figure 4: Evolution of the Landweber-Kaczmarz method for one single measurement $(N=1)$. The corresponding source is shown in Figure 3 (a). 

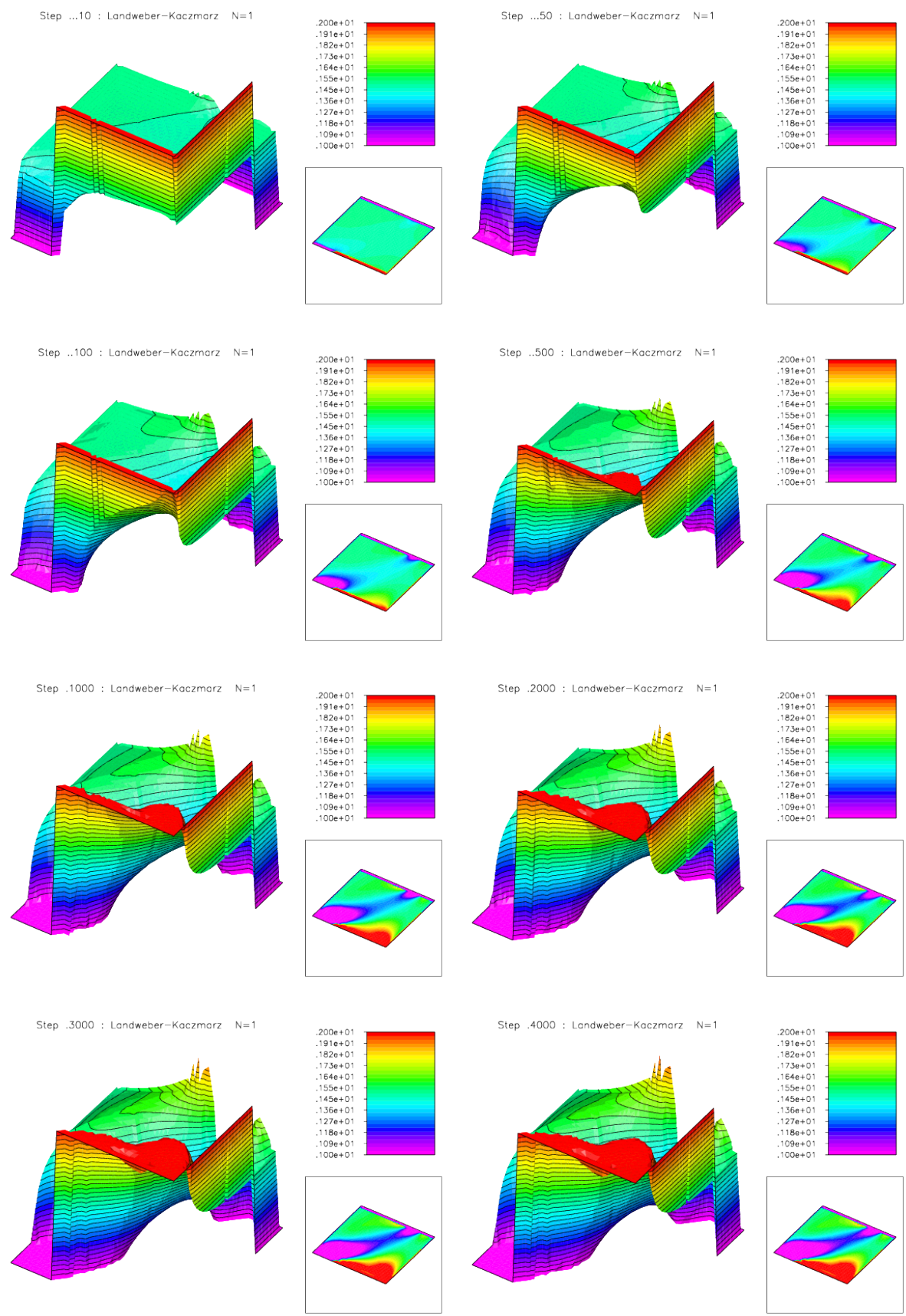

Figure 5: Evolution of the Landweber-Kaczmarz method for one single measurement $(N=1)$. The corresponding source is shown in Figure $3(\mathrm{~b})$. 

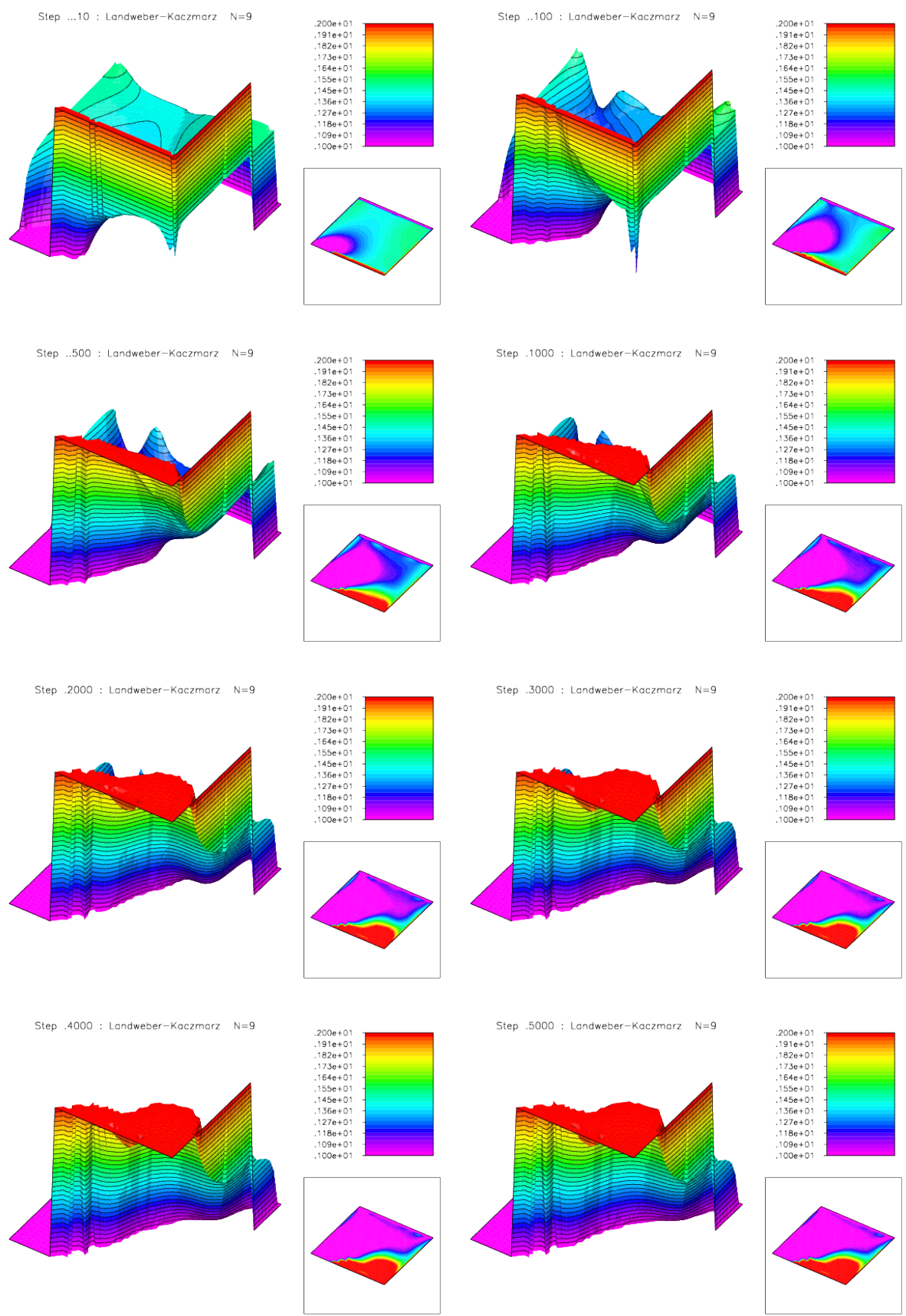

Figure 6: Evolution of the Landweber-Kaczmarz method for $N=9$ and exact data. 

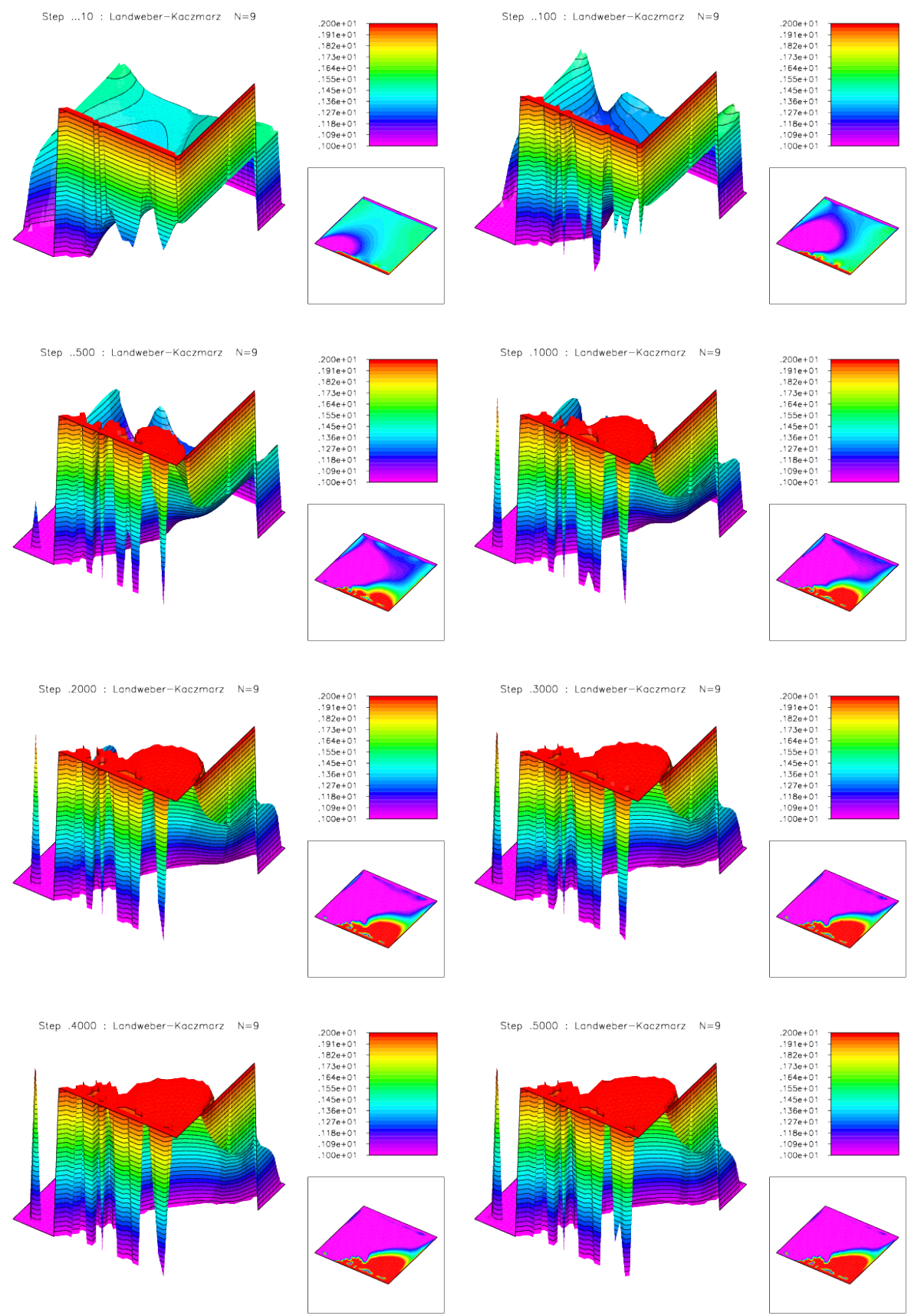

Figure 7: Evolution of the Landweber-Kaczmarz method for $N=9$ and noisy data. Noise level of $10 \%$. 